\title{
Isospin effects and symmetry energy studies with INDRA
}

\author{
G. Ademard ${ }^{1}$, B. Borderie ${ }^{1}$, A. Chbihi ${ }^{2}$, O. Lopez ${ }^{3}$, P. Napolitani ${ }^{1}$, M. F. Rivet ${ }^{1}$, M. Boisjoli ${ }^{2,4}$, E. Bonnet ${ }^{2}$, \\ R. Bougault ${ }^{3}$, J. D. Frankland ${ }^{2}$, E. Galichet ${ }^{1,5}$, D. Guinet ${ }^{6}$, M. Kabtoul $^{3}$, G. Lehaut ${ }^{3}$, P. Lautesse ${ }^{7}$, M. La Commara $^{8}$,

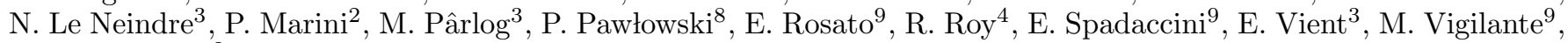 \\ J. P. Wieleczko ${ }^{2}$ \\ , and (INDRA Collaboration) \\ 1 Institut de Physique Nucléaire, CNRS-IN2P3 and Université Paris-Sud 11, F-91406 Orsay cedex, France \\ 2 GANIL, CEA-DSM/CNRS-IN2P3, B.P. 5027, F-14076 Caen cedex, France. \\ 3 LPC Caen, ENSICAEN, Université de Caen, CNRS-IN2P3, F-14050 Caen cedex, France. \\ 4 Université Laval, Québec, G1V 0A6 Canada. \\ 5 Conservatoire National des Arts et Métiers, F-75141 Paris cedex 03, France. \\ 6 Institut de Physique Nucléaire, UCBL, Université de Lyon, CNRS-IN2P3, F-69622 Villeurbanne cedex, France. \\ 7 S2HEP (EA4148), UCBL/ENSL, Université de Lyon, Villeurbanne, France. \\ 8 IFJ-PAN, 31-342 Kraków, Poland \\ 9 Dipartimento di Scienze Fisiche e Sezione INFN, Universitá di Napoli "Federico II", I80126 Napoli, Italy.
}

October 3, 2018

\begin{abstract}
The equation of state of asymmetric nuclear matter is still controversial, as predictions at subsaturation as well as above normal density widely diverge. We discuss several experimental results measured in heavy-ion collisions with the INDRA array in the incident energy range 5-80 $\mathrm{AMeV}$. In particular an estimate of the density dependence of the symmetry energy is derived from isospin diffusion results compared with a transport code: the potential part of the symmetry energy linearly increases with the density. We demonstrate that isospin equilibrium is reached in mid-central collisions for the two reactions $\mathrm{Ni}+\mathrm{Au}$ at $52 \mathrm{AMeV}$ and $\mathrm{Xe}+\mathrm{Sn}$ at $32 \mathrm{AMeV}$. New possible variables and an improved modelization to investigate symmetry energy are discussed.
\end{abstract}

PACS. 2 5.70.Pq -2 4.60.Ky

\section{Introduction}

Heavy-ion collisions are used as an experimental probe for isospin effects related to the equation of state of isospinasymmetric nuclear matter. At the same time, microscopic models are worked out in order to incorporate and test isospin effects in the description of nuclear processes. The physical picture and the corresponding modelling requirements depend on the range of incident energies they apply to. Several different forms are suggested for the isospin contribution to the equation of state, in particular as a consequence of many alternative descriptions of the density dependence of the symmetry energy [1]. The difference between these forms stands out dramatically when the saturation density is exceeded; since such condition can be achieved at large bombarding energies, the corresponding modelling approach should be built by taking into account, in the isovector channel, effects like the momentum dependence and the hadron effective mass splitting 2 .

The route taken by the INDRA collaboration is to investigate the subsaturation-density domain, explored at

Correspondence to: rivet@ipno.in2p3.fr
Fermi energies. The specific interest of this choice, although the sensitivity to the density dependence of the symmetry energy is reduced (implying to take into account also finer effects like the secondary decay), is that in this energy regime isospin effects can be searched in relation with transport observables, like diffusion and migration processes [3 5], or with cluster-correlation properties and fragment observables, like phase-transition features [6, bimodalities 7, 8, spinodal effects and distillation processes [9]. This is the physical landscape addressed by INDRA.

The $4 \pi$ multidetector INDRA, which is described in detail in 10, 11, was thus used to reveal $\mathrm{N} / \mathrm{Z}$ effects in heavy-ion reactions connected to the knowledge of symmetry energy entering the asymmetric nuclear equation of state $3,12,13$. In terms of detection INDRA possesses excellent performance: geometrical efficiency of $90 \%$, rather low detection and identification thresholds (see Fig. 1 of ref. [14), accurate charged particle and fragment identifications, energy measurements with an accuracy of $4 \%$. Further details can be found in Refs. [15 17. The global quality of detection was then used to, in particular, per- 
form data selections and reconstruct excited nuclear systems to compare with simulations over large ranges of impact parameters.

The paper is organized as follows. Results obtained for central collisions are evidenced in section 2 and a new variable to investigate symmetry energy effects is proposed. In section 3, semiperipheral collisions are discussed and evolutions of observables with the impact parameter are compared to theoretical simulations to derive information on the potential part of the symmetry energy term from isospin diffusion. Average stopping for central collisions with different $\mathrm{N} / \mathrm{Z}$ entrance channels are presented in section 4. Section 5 is devoted to studies in progress related to projectile fragmentation at Fermi energy, fusion reactions at low energies with different $\mathrm{N} / \mathrm{Z}$ and comparison of data with a new transport code. Some foreseen studies are described in section 6. Conclusions are drawn in section 7. Finally in the appendix we gather the different experimental selections made for the presented data, some of them being specific to the INDRA array.

\section{Isospin effects in central collisions}

In this section we describe isospin effects observed in quasifusion reactions. First constraints on the symmetry term of the EOS could be obtained by studying nuclear reactions with judiciously chosen projectile-target couples. We bombarded ${ }^{112,124} \mathrm{Sn}$ targets with ${ }^{124,136}$ Xe projectiles accelerated at 32 and $45 \mathrm{AMeV}$ by the GANIL facility; the four possible configurations were studied at $32 \mathrm{AMeV}$ while ${ }^{124} \mathrm{Xe}+{ }^{124} \mathrm{Sn}$ was not at $45 \mathrm{AMeV}$. The ${ }^{124} \mathrm{Xe}+{ }^{124} \mathrm{Sn}$ and ${ }^{136} \mathrm{Xe}+{ }^{112} \mathrm{Sn}$ systems have the same combined isospin $(\mathrm{N} / \mathrm{Z}=1.385)$, while the isospin gradient between projectile and target is 1.5 times larger for the second one.

Central collisions were selected as explained in section A.1. Results concerning the LCP and fragment $(\mathrm{Z} \geq 5)$ multiplicities, as well as the centre of mass average fragment kinetic energies, were published in [18. However, as mentioned in that reference, we collected a very large number of events; it thus becomes possible to extrapolate the values of different variables to those corresponding to a perfect detection of charges, $\mathrm{Z}_{t o t}=104$ (the procedure is described in the appendix A.1). This will facilitate the comparison with simulations, avoiding the filtering of the calculated events. We observed that the widths of the multiplicity distributions are not modified by the degree of completeness, whereas those of the $\mathrm{Z}_{\text {bound }}$ variables decrease for more complete events. We verified that the charge and angular distributions of charged products were not modified when strengthening the event completeness.

Obviously the multiplicities obtained with this procedure are larger than those published in 18, but the trends observed as a function of the total system isospins are not modified, as displayed in fig. 1. $M_{l c p}$ linearly decreases with increasing isospin, with a steeper slope at the higher energy, whereas $M_{\text {frag }}$ linearly increases, with equal slopes at 32 and $45 \mathrm{AMeV}$. At $32 \mathrm{AMeV}$, we measured the two mixed systems, ${ }^{124} \mathrm{Xe}+{ }^{124} \mathrm{Sn}$ and ${ }^{136} \mathrm{Xe}+{ }^{112} \mathrm{Sn}$; we
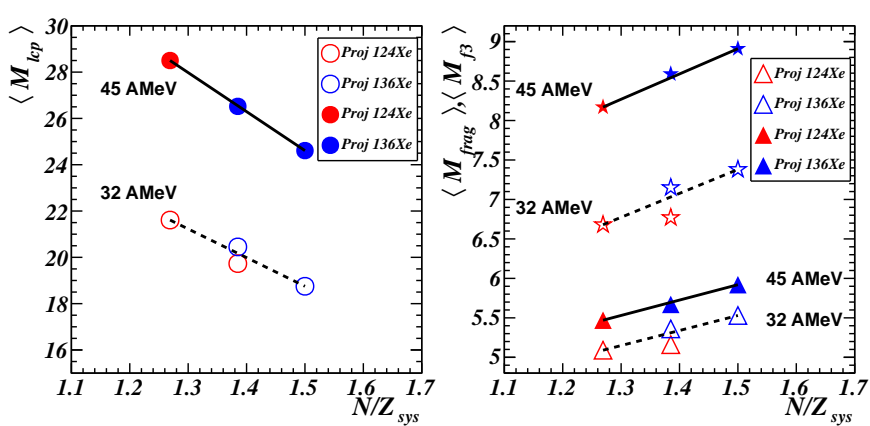

Fig. 1. (Colour on line) Left: evolution of the mean multiplicities of LCP measured for quasi-fusion reactions $v s$ the N/Z of the different $\mathrm{Xe}+\mathrm{Sn}$ total systems at 32 and $45 \mathrm{AMeV}$. Right: same for mean fragment multiplicities, $M_{f 3}(\mathrm{Z} \geq 3$, stars) and $M_{\text {frag }}(\mathrm{Z} \geq 5$, triangles).

observe that both LCP and fragment multiplicities little depend on the entrance channel mass asymmetry.

In short we observed that in multifragmentation of quasi-fused systems, more fragments, and less LCP, are emitted when the system grows neutron-richer. In previous works the increase of fragment multiplicity was attributed to phase-space effects [19]. We underlined in [18] that the $10 \%$ difference measured both at 32 and $45 \mathrm{AMeV}$ between the lightest and the heaviest systems correspond to the mass increase between them. It recalls the scaling law observed in 20 and attributed to volume instabilities as the origin of the multifragmentation phenomenon (spinodal decomposition). We tested this hypothesis by comparing our data to a stochastic transport code.

\subsection{The Stochastic Mean Field model}

At Fermi energy and below an appropriate description of the density profile in phase space demands an accurate description of mean-field properties and a precise treatment of the Pauli blocking. At these energies, even if the early topology of the system may suggest a final fragmented configuration, transport mechanisms driven by density gradients (isospin migration) or by isospin gradients (isospin diffusion) may still progress up to few hundred $\mathrm{fm} / c$ and affect the freeze-out configuration. In order to apply efficiently to such situation a one-body description should therefore be preferred, as it is fully adapted to follow the bulk behaviours for a long interval of time, corresponding with the complete duration of the process. In this framework, central and semicentral collisions are particularly interesting: they allow to explore the largest span in nuclear density and to access very small values compatibles with the disassembling of the system. According to experimental and theoretical studies 21, 22, at bombarding energies around $30 \mathrm{AMeV}$, for a system of average size like $\mathrm{Xe}+\mathrm{Sn}$, the dynamics of the system should be largely determined by spinodal behaviours: this is actually a consequence of the form of the equation of state and of the properties of the mean field which stands out when approaching the low-energy threshold of multifragmentation. 
The above phenomenology is well described using the Boltzmann-Langevin (BL) equation that describes the evolution of the semiclassical one-body distribution function $f(\mathbf{r}, \mathbf{p}, t)$ submitted to the effective Hamiltonian $H[f]$ and the residual interaction; this latter, expressed in terms of the one-body distribution function $f$ includes the average Boltzmann collision integral $\bar{I}[f]$, and the fluctuating term $\delta I[f]:$

$$
\dot{f}=\partial_{t} f-\{H[f], f\}=\bar{I}[f]+\delta I[f] .
$$

Various approximate treatments of the BL equation have been developed. The Stochastic Mean Field model, SMF, uses the following method [23]: within the assumption of local thermal equilibrium, the stochastic term of Eq. 1 . $\delta I[f]$, builds kinetic equilibrium fluctuations typical of a Fermi gas, which are projected on density fluctuations in the coordinate space. The fluctuations so introduced are amplified by the unstable mean field, and the dynamics is essentially driven by the propagation of mean field instabilities [24].

We compared the results of this section and of the next one with the SMF model. The free nucleon-nucleon cross section with its angular, energy and isospin dependence is used. We take a soft isoscalar equation of state, with a compressibility modulus $\mathrm{K}=200 \mathrm{MeV}$. Two different parameterizations of the symmetry energy are used; in the "asystiff" one the potential term increases linearly with the density whereas the "asysoft" one is taken from the $\mathrm{SKM}^{*}$ Skyrme formulation [25].

The symmetry term of the EOS, $E_{\text {sym }} / A$, is often parameterized as

$$
\frac{E_{\text {sym }}}{A}(\rho)=\frac{C_{s, k}}{2}\left(\frac{\rho}{\rho_{0}}\right)^{2 / 3}+\frac{C_{s, p}}{2}\left(\frac{\rho}{\rho_{0}}\right)^{\gamma}
$$

where $\rho_{0}$ is the nuclear saturation density. The first term is kinetic, coming from Pauli correlations; the second term is the potential part, that carries the isovector properties of the effective nuclear interaction. The value of the $\gamma$ exponent, valid close to $\rho_{0}$, determines whether the equation is "asystiff" $(\gamma \geq 1$, potential term continuously increasing with $\rho)$ or "asysoft" $(\gamma<1$, potential term presenting a maximum between $\rho_{0}$ and $\left.2 \rho_{0}\right)$.

A second order expansion around $\rho_{0}$ of the potential term of the symmetry energy reads:

$$
\frac{E_{\text {sym }}}{A}(\rho)=S_{0}+\frac{L}{3}\left(\frac{\rho-\rho_{0}}{\rho_{0}}\right)+\frac{K_{\text {sym }}}{18}\left(\frac{\rho-\rho_{0}}{\rho_{0}}\right)^{2}
$$

In the SMF framework values of $\mathrm{L} \geq 75$ correspond to an asystiff $E_{\text {sym }}[3]$.

At any step of the calculation fragments are recognized by applying a coalescence procedure to the one-body density, connecting nearby cells in which the density is larger than a cut-off value, taken equal to $\rho_{0} / 5$ ("liquid phase"). We have shown in [26] that, at $300 \mathrm{fm} / c$, the fragment multiplicity is independent of the exact value of the cut-off density. The remaining early emitted nucleons constitute the "gas phase". The fragment phase space configuration at $300 \mathrm{fm} / c$ is injected in the SIMON code 27] which performs the secondary decay during the propagation of all products under the Coulomb field, thus preserving spacetime correlations.

\subsection{Multiplicities and $Z_{\text {bound }}$ from SMF}

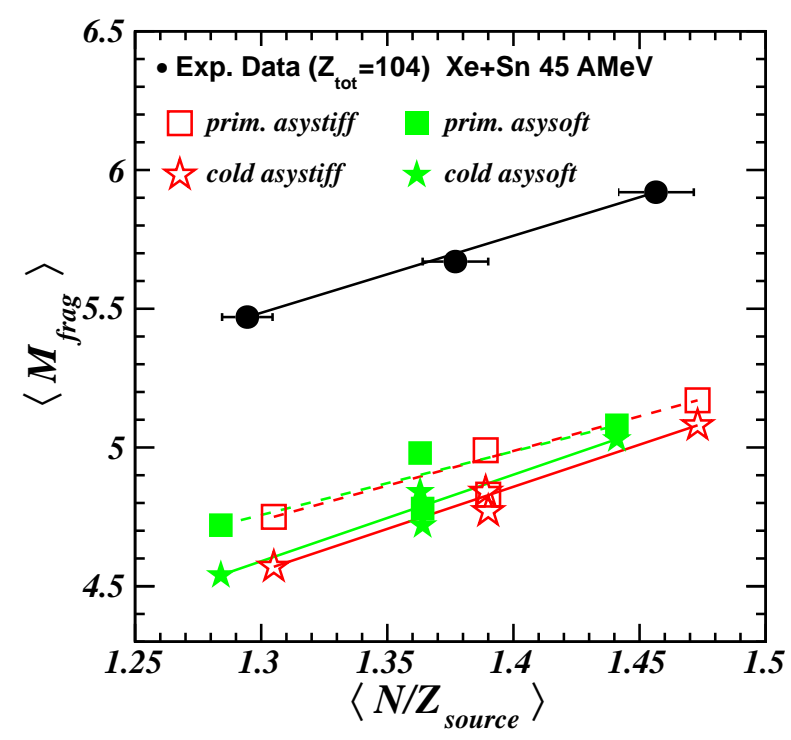

Fig. 2. (Colour on line) Average fragment $(\mathrm{Z} \geq 5)$ multiplicities, for the different systems at $45 \mathrm{AMeV}$ : experimental values extrapolated to $Z_{t o t}=104$ (circles, the line is to guide the eye); calculated values for primary (squares) and cold fragments (stars), are plotted vs the source N/Z. Asystiff EOS: open symbols, asysoft EOS: filled symbols. In all cases statistical error bars are smaller than the symbol sizes.

For head-on collisions between Xe and Sn at $45 \mathrm{AMeV}$ SMF predicts the formation of a single source which subsequently breaks into several fragments. The fragment excitation energy at $\mathrm{t}=300 \mathrm{fm} / c$ is around $3.3 \mathrm{AMeV}$, in good agreement with experimental evaluations [28, 29]; we also verified that the experimental charge distributions are reasonably accounted for by the simulation. Conversely at $32 \mathrm{AMeV}$ the systems do not multifragment and lead to an evaporation residue, because the treatment of the fluctuation mechanism is only described in average. An improvement have therefore to be worked out on this aspect (see section 5.3).

We present in figure 2 the measured and calculated fragment multiplicities at $45 \mathrm{AMeV}$. Results from the simulations are plotted at the $\mathrm{N} / \mathrm{Z}$ value of the fragmenting source, at $\mathrm{t}=120 \mathrm{fm} / \mathrm{c}$, after preequilibrium emission. Note that the $\mathrm{N} / \mathrm{Z}$ range is reduced with respect to that covered by the initial systems. Indeed for both EOS the $n / p$ ratio of preequilibrium is larger than that of the total system for ${ }^{136} \mathrm{Xe}+{ }^{124} \mathrm{Sn}$, whereas it is smaller for ${ }^{124} \mathrm{Xe}+{ }^{112} \mathrm{Sn}$; consequently the source $\mathrm{N} / \mathrm{Z}$ varies in reverse direction. For the mixed systems, $\mathrm{n} / \mathrm{p}$ and the source $\mathrm{N} / \mathrm{Z}$ are identical to the system one in the asystiff case. In all cases preequilibrium emission is more neutron-rich - thus the source 

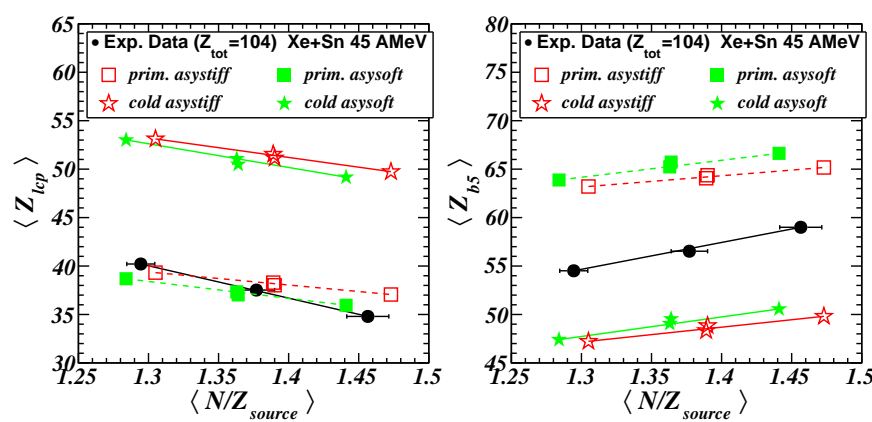

Fig. 3. (Colour on line) Left: evolution of the mean charge bound in LCP measured for quasi-fusion reactions vs the N/Z of the different $\mathrm{Xe}+\mathrm{Sn}$ total systems at $45 \mathrm{AMeV}$. Right: same for mean charge bound in fragments, $Z_{b 5}$, Symbols as in fig. 2

less neutron-rich - in the asysoft case. In figures 2 and 3 experimental data are drawn at the average $N / Z$ of the asysoft and asystiff sources. We show only $M_{\text {frag }}$ and not $M_{f 3}$ because the former is little modified by the cooling stage, as appears from the figure, comparing squares and stars, whereas the production of $\mathrm{Li}$ and $\mathrm{Be}$ is increases during cooling. The difference between primary and final multiplicities is even smaller for the neutron-rich system. We note that the simulations lead to primary fragment multiplicities increasing with the neutron richness of the source: the observed effect comes from symmetry energy, and is only slightly accentuated by evaporation. However the calculated final multiplicitie are smaller than the experimental one by about 1 unit (15\%). To get a deeper insight into the phenomenon, we turn to $Z_{\text {bound }}$ variables. Indeed while the LCP multiplicity does not make sense in the simulation, because essentially nucleons are produced in the preequilibrium and multifragmentation phases, the charge bound in LCP is meaningful. In figure 3 we present the evolution of $Z_{l c p}$ and $Z_{b 5}$ with the source N/Z. We observe firstly that the experimental trend is reproduced by the simulation already before de-excitation, confirming that we are dealing with a symmetry energy effect. $Z_{l c p}$ at that stage is already equal to the measured one, which indicates that the preequilibrium emission is too abundant in the model. And indeed when we compare the data with the final simulated values, we have too much charge bound in lcp and not enough in fragments.

The difference between the measured $Z_{b 5}$ values for the neutron-richer and the neutron-poorer systems is about 5.5 units $(9.8 \%)$. It is smaller in the simulation, 2.6 units $(5.3 \%)$ in the asystiff case and $3.2(6.5 \%)$ with the asysoft EOS. This observation, although it does not allow to make a choice between the asy-EOS, indicates that the $Z_{b 5}$ variable is more promising than the fragment multiplicity for constraining the EOS.

Another observable shown in 18 is the centre of mass average kinetic energy of the fragments. At both incident energies we observed that for each $\mathrm{Z}$ value it slightly increases with the $\mathrm{N} / \mathrm{Z}$ of the system. Assuming that the Coulomb and thermal components of the kinetic energy are identical for all $\mathrm{Xe}+\mathrm{Sn}$ systems at a given incident energy, we induce that the expansion component is larger, because heavier primary isotopes are produced when more neutrons are available in the system. The fragment kinetic energies obtained in the SMF simulations are far below the experimental ones, due to the too large preequilibrium emission (see section 5.3).

\subsection{Summary}

Comparing $\mathrm{Xe}+\mathrm{Sn}$ quasi-fusion reactions for different projectile-target couples, we have observed experimentally that more fragments, with larger kinetic energies, are produced when the system is neutron-richer. The SMF model reproduces these trends, before the cooling stage, meaning that we are dealing with effects due to the interaction, more precisely to the symmetry energy. The charge bound in fragments appears as an interesting isospin sensitive variable, for which the simulation better reproduces the trend (slope) with the asy-soft EOS.

\section{Symmetry energy from isospin diffusion}

Isospin transport during semi-peripheral collisions between projectiles and targets differing by their isospin content is one of the observables sensitive to the symmetry term of the nuclear equation of state. Around the Fermi energy the isospin content of the quasi-projectile (QP) and the quasi-target $(\mathrm{QT})$ is determined by the interplay between fast particle emission during the overlap between the incident partners and the transfer of nucleons through the neck which develops between them 30. Assuming local thermal equilibrium the isospin transport coefficients can be derived within the hydrodynamic limit; the proton and neutron exchange is governed by the gradients of the respective chemical potentials and the current of the two species comprises two terms. One is the isospin migration arising from density gradient. The second one is the isospin diffusion due to the different isospin content of the reaction partners [2, 3]. The difference of the neutron and proton currents is directly connected to the symmetry energy: the difference of the migration coefficients is proportional to the isospin value times the slope of the symmetry energy versus density whereas that of the diffusion coefficients is proportional to the density times the absolute value of the symmetry energy.

It comes from the above considerations that even for a symmetric system (identical projectile and target) one may observe some isospin content evolution of the QP and QT with the incident energy and the impact parameter, due to preequilibrium emission and isospin migration.

\subsection{Isospin transport}

The INDRA collaboration studied isospin transport by looking at the quasi-projectile isospin content, as both detection and isotopic identification are better in the forward part of the INDRA array. Isospin transport was studied 
as a function of the impact parameter (parameterized either via the dissipated energy or via the transverse energy of the light charged particles, see section A.2. Information on the stiffness of the symmetry energy was derived through a comparison of the experimental data to the results of the Stochastic Mean Field transport model briefly described in section 2.1

The INDRA collaboration studied isospin transport for different systems. The reader is sent to the published papers for details of the experiments; selections are described in section A.2

\subsubsection{Ni induced reactions}

In a first experiment a ${ }^{58} \mathrm{Ni}$ projectile accelerated at 52 and $74 A \mathrm{MeV}$ by the GANIL facility bombarded ${ }^{58} \mathrm{Ni}$ and ${ }^{197} \mathrm{Au}$ targets 3133 . The QP isospin variation was followed thanks to the variable

$$
(<\mathrm{N}>/<\mathrm{Z}>)_{\mathrm{CP}}=\sum_{N_{\text {evts }}} \sum_{\nu} N_{\nu} / \sum_{N_{\text {evts }}} \sum_{\nu} P_{\nu}
$$

where $N_{\nu}$ and $P_{\nu}$ are respectively the numbers of neutrons and protons bound in particle $\nu, \nu$ being $\mathrm{d}, \mathrm{t},{ }^{3} \mathrm{He},{ }^{4} \mathrm{He}$, ${ }^{6} \mathrm{He},{ }^{6} \mathrm{Li},{ }^{7} \mathrm{Li},{ }^{8} \mathrm{Li},{ }^{9} \mathrm{Li},{ }^{7} \mathrm{Be},{ }^{9} \mathrm{Be},{ }^{10} \mathrm{Be}$; free protons are excluded. $N_{\text {evts }}$ is the number of events contained in the dissipated energy bin considered. The variable $(N / Z)_{C P}$ was calculated twice: first considering particles forward emitted in the nucleon-nucleon frame $\left(\mathrm{V}_{\text {particle }}>\mathrm{V}_{\text {proj }}^{\text {lab }} / 2\right)$, and secondly keeping only particles forward emitted in the QP frame $\left(\mathrm{V}_{\text {particle }}>\mathrm{V}_{\mathrm{QP}}^{\mathrm{rec}}\right)$. In the first case mid-rapidity particles and those coming from the hot QP de-excitation are considered. In the second case, only the latter type of particles are kept.

Figure 4 displays the evolution of $(N / Z)_{C P}$ with the violence of the collision. Open points show the values obtained forward in the $\mathrm{NN}$ frame. In this case we mix mid-rapidity particles and those coming from the QP deexcitation. For the $\mathrm{Ni}+\mathrm{Ni}$ system at both incident energies, $(N / Z)_{C P}$ varies by at most $1.5 \%$ when dissipation increases. This is the expected behaviour for this symmetric system where $\mathrm{N} / \mathrm{Z}$ is only modified by pre-equilibrium emission. For the $\mathrm{Ni}+\mathrm{Au}$ system the isospin ratio is higher than that of the $\mathrm{Ni}+\mathrm{Ni}$ system whatever the dissipated energy. At small dissipation this could arise from the neutron skin of the Au target and/or from the mid-rapidity particles included in our quasi-projectile selection, which are more neutron rich 34, 35. This result is a first indication of isospin diffusion. Then $(N / Z)_{C P}$ presents a significant increase with dissipation and reaches higher values at $52 \mathrm{AMeV}$, while the trend is flatter at $74 A \mathrm{MeV}$. This may be interpreted as a progressive isospin diffusion when collisions become more central, in connection with a larger overlap of the reaction partners and thus a longer interaction time.

The close points in fig 4 are related to the values of $(N / Z)_{C P}$ forward in the QP frame. They are in all cases smaller than the previous ones, and for $\mathrm{Ni}+\mathrm{Au}$ at both energies, they grow faster with dissipation. This is because
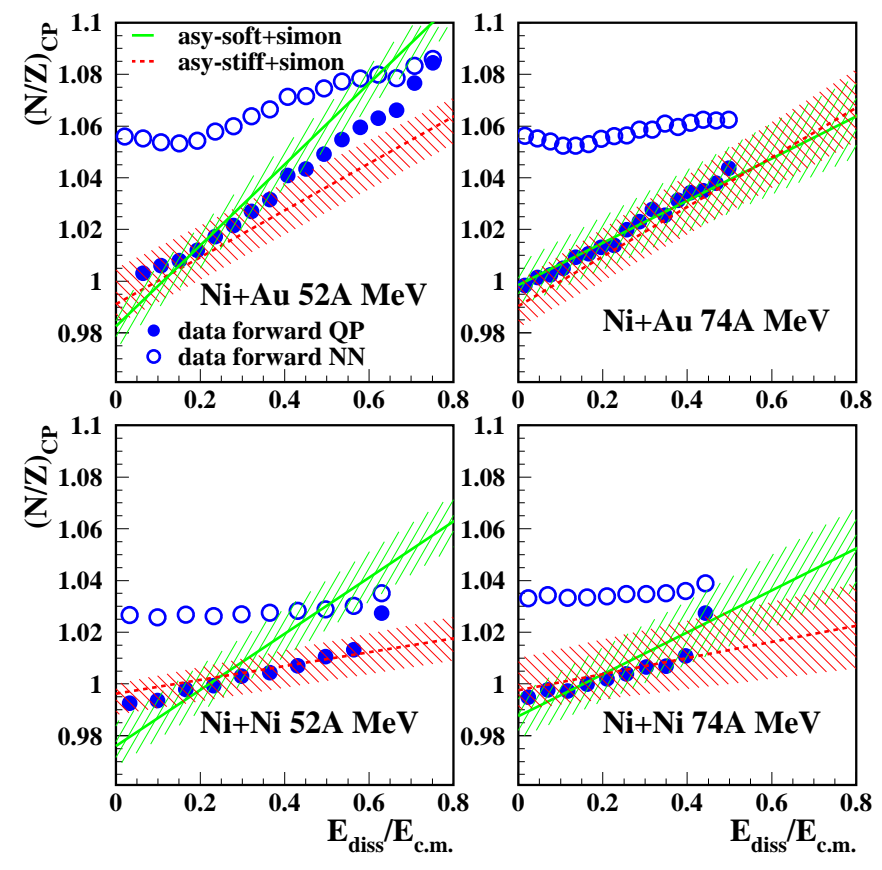

Fig. 4. (Colour on line) Isospin ratio of complex particles, $(N / Z)_{C P}$, vs the dissipated energy. Circles correspond to experimental data: open, forward in NN frame, close, forward in QP frame. Error bars are within the size of the symbols. Dotted and solid lines correspond respectively to the asystiff and asysoft parametrizations, the hatched zones give error bars from simulations. From. 32

the neutron-rich mid-rapidity particles are no longer included; indeed it is known that their isospin content is independent of the violence of the collision. 34

The values of $(N / Z)_{C P}$ forward in the QP frame are compared with the results of the SMF simulations, after de-excitation of the hot $\mathrm{QP}$, displayed in the figure by the lines and the hatched zones. A first result worth mentioning is that the chemical composition $(\mathrm{N} / \mathrm{Z})$ of the quasiprojectile forward emission appears as a very good representation of the composition of the entire quasi-projectile source. Such an observation seems to validate a posteriori the selection frequently used to characterize the QP de-excitation properties. When looking globally at the results for the four cases treated here, the agreement is better when the asy-stiff EOS is used, i.e. a linear increase of the potential term of the symmetry energy around normal density. Note however that for $\mathrm{Ni}+\mathrm{Au}$ at $52 \mathrm{AMeV}$, where isospin transport effects are dominant, the close points lie in between the simulated results with the two EOS. This observation allows us to put an error bar on our result, expressed as $\gamma=1 \pm 0.2$ (see Eq. 2), or $\mathrm{L}=75 \pm 25 \mathrm{MeV}$ (Eq.3).

\subsubsection{Xe induced reactions}

A second study used $32 \mathrm{AMeV}{ }^{124,136} \mathrm{Xe}$ projectiles impinging on ${ }^{112,124} \mathrm{Sn}$, as described in section 2 . It is interesting to note that the total systems obtained in the $\mathrm{Ni}+\mathrm{Au}$ reaction at $52 \mathrm{AMeV}$, and the ${ }^{124} \mathrm{Xe}+{ }^{124} \mathrm{Sn}$ and 


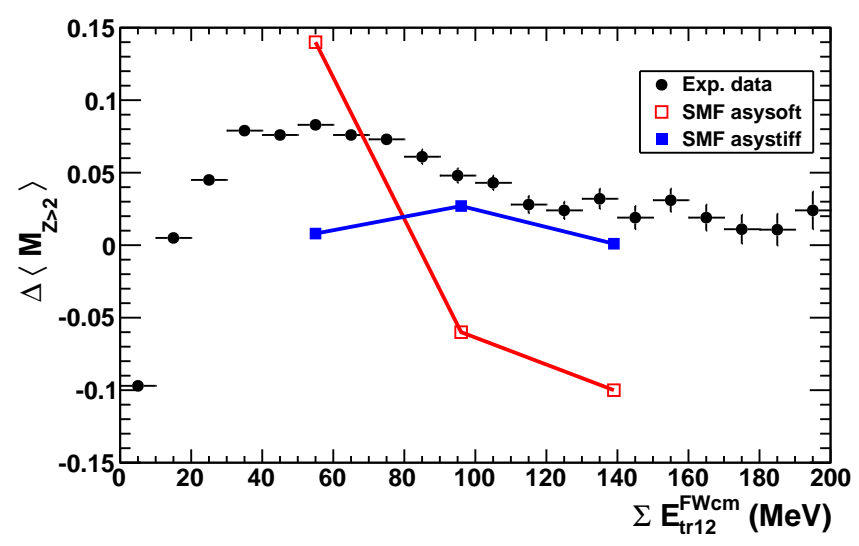

Fig. 5. (Colour on line) Difference of the fragment $(Z>2)$ multiplicities, for the systems, ${ }^{136} \mathrm{Xe}+{ }^{112} \mathrm{Sn}$ and ${ }^{124} \mathrm{Xe}+{ }^{124} \mathrm{Sn}$, vs the transverse energy of light charged particles detected in the forward $\mathrm{cm}$ hemisphere. Close points show the experimental data with statistical error bars. Open squares correspond to the asysoft SMF simulation, close squares to the asystiff one. For simulations, the difference is calculated for primary fragments, before de-excitation. From. 36, 37.

${ }^{136} \mathrm{Xe}+{ }^{112} \mathrm{Sn}$ reactions at $32 \mathrm{AMeV}$, are very similar and have the same $\mathrm{N} / \mathrm{Z}$. The available centre of mass energies per nucleon differ by $1 A \mathrm{MeV}$, being $\sim 9 \mathrm{MeV}$ for $\mathrm{Ni}+\mathrm{Au}$ at $52 \mathrm{AMeV}$ and $\sim 8 \mathrm{MeV} \mathrm{Xe+Sn}$ at $32 \mathrm{AMeV}$.

The chosen isospin sensitive variable is the fragment multiplicity difference between the ${ }^{136} \mathrm{Xe}+{ }^{112} \mathrm{Sn}$ and ${ }^{124} \mathrm{Xe}+{ }^{124} \mathrm{Sn}$ systems:

$$
\Delta M_{Z>2}=M_{Z>2}^{136} X e+{ }^{112} S n-M_{Z>2}^{124} X e+{ }^{124} S n
$$

followed as a function of the violence of the collision. The impact parameter scale is given by the transverse energy of the light charged particles: values $E_{t r 12}^{F W c m}$ of 50,100 and $140 \mathrm{MeV}$ correspond to experimental impact parameters $\mathrm{b}=8,6$ and $4 \mathrm{fm}$ respectively. Only products detected in the forward centre of mass hemisphere are considered. Quasi-fusion events are removed by means of the variable $V_{\text {bigiso }}$ (see section A.1.

The measured $M_{Z>2}$ is the sum of fragments coming from the QP de-excitation and from mid-rapidity. For $E_{t r 12}^{F W c m}>100 \mathrm{MeV}$ isospin equilibrium is reached (see section 3.2, thus the QP de-excitation properties are the same, in particular the multiplicities of the emitted fragments. Therefore $\Delta M_{Z>2}$ reduces to the difference between mid-rapidity multiplicities. Assuming that these multiplicities are not modified by the de-excitation stage, the measured $\Delta M_{Z>2}$ can be directly compared to the same difference obtained in SMF simulations for primary fragments. This avoids to resort to a de-excitation code. Looking at fig. 5, one observes that the experimental value of $\Delta M_{Z>2}$ levels-off above $E_{t 12}^{F W c m}=110 \mathrm{MeV}$. If we now compare with the simulated values, it appears that the asy-soft case does not follow the experimental trend, wherea the asystiff calculation well matches the data for $E_{t r 12}^{F W c m}>$ $100 \mathrm{MeV}$, or $b<6 \mathrm{fm}$. For more peripheral collisions, the above assumptions do not hold because isospin equilibrium is not reached, thus simulations and data diverge.

\subsection{Isospin equilibration}

Ultimately isospin transport tends to equilibrate the isospin content of the two reaction partners. Whether equilibrium is reached or not depends on the relative values of the characteristic time of the mode, and of the reaction time (namely when the QP and QT separate). In deep inelastic reactions at energies close to the Coulomb barrier, the $\mathrm{N} / \mathrm{Z}$ equilibrium is rapidly reached, within $\sim 1-2 \times 10^{-22} \mathrm{~s}[38,39$. In that case isospin equilibration is governed by a large amplitude dipole collective motion 2. Around Fermi energy the experimental situation is not so clear. The first experiments in the 80 's studied $\mathrm{Ar}$ and $\mathrm{Kr}$ induced very peripheral reactions, in which isospin transport was visible but equilibrium was clearly not reached [40]. In most of the recent papers, data obtained at these energies, with some selection, were compared to transport calculations performed at a given, supposedly matching impact parameter. At those energies, as said above, the chemical potential gradient governs isospin exchange.

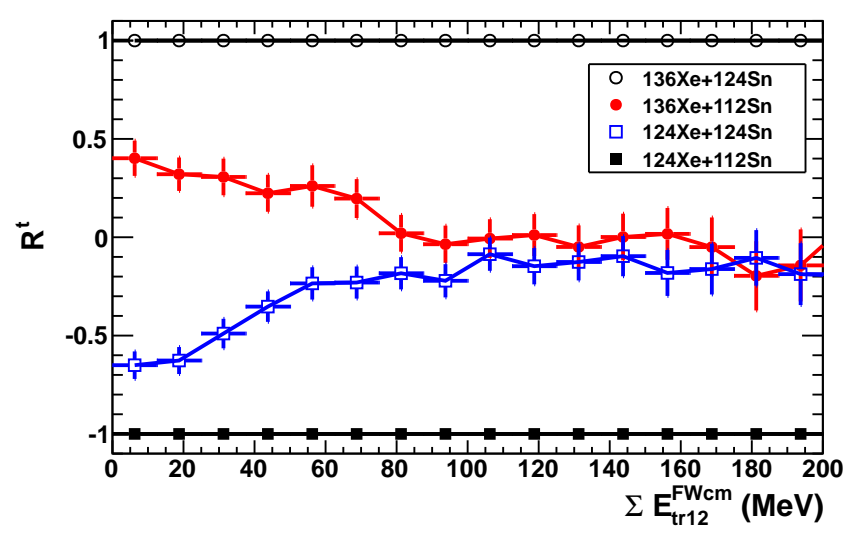

Fig. 6. Isospin transport ratio calculated for forward triton multiplicity measured for the four $\mathrm{Xe}+\mathrm{Sn}$ systems at $32 \mathrm{AMeV}$ as a function of the dissipated energy. From. 37.

As demonstrated in subsection 3.1, we have the possibility to follow isospin transport from peripheral to almost central collisions, and to anchor rather firmly the experimental and calculated impact parameters. We have arguments to state, from the experimental data alone, that in two cases isospin equilibrium is reached. Looking at the top-left panel of fig. 4, we observe that at high dissipation $\left(E_{\text {diss }} / E_{\text {c.m. }}>0\right.$.7, i.e. $b<5$ fm from fig. 2) the values of $(N / Z)_{C P}$ are the same, whether it is calculated with or without mid-rapidity particles. This is a strong indication of isospin equilibrium for the ${ }^{58} \mathrm{Ni}+{ }^{197} \mathrm{Au}$ reaction at $52 \mathrm{AMeV}$. As far as the $\mathrm{Xe}+\mathrm{Sn}$ systems are concerned, se show in fig. 6 the isospin transport ratio [4]

$$
R_{P, T}^{x}=\frac{2\left(x^{M}-x^{e q}\right)}{\left(x^{H}-x^{L}\right)} \text { with } x^{e q}=\left(x^{H}+x^{L}\right) / 2
$$


the index $\mathrm{H}$ refers to the n-rich system ${ }^{136} \mathrm{Xe}+{ }^{124} \mathrm{Sn}$ and $\mathrm{L}$ to the $\mathrm{n}$-poor ${ }^{124} \mathrm{Xe}+{ }^{112} \mathrm{Sn}$ system, $\mathrm{M}$ to the mixed reactions ${ }^{124} \mathrm{Xe}+{ }^{124} \mathrm{Sn}$ and ${ }^{136} \mathrm{Xe}+{ }^{112} \mathrm{Sn}$. The chosen isospin sensitive variable is the triton multiplicity in the forward centre of mass hemisphere. The evolution of $R^{t}$ with dissipation is displayed in fig. 6. We observe that isospin equilibrium is reached above a transverse LCP energy of 100 $\mathrm{MeV}$. The same conclusion is obtained if the variable $\mathrm{x}$ is the fragment multiplicity [37].

Finally we studied almost symmetric and a very asymmetric reactions for which the composite systems have charges 104 and 107 and a value of N/Z of 1.38, at available energies 8-9 $A \mathrm{MeV}$. We observed experimentally that isospin equilibrium is reached for rather high dissipation. To go further we turn to the SMF simulations. For $\mathrm{Ni}+\mathrm{Au}$ at $52 \mathrm{AMeV}$, isospin equilibrium is predicted for an impact parameter $\mathrm{b} \leq 4 \mathrm{fm} 32$. The corresponding reaction time is $4 \times 10^{-22} \mathrm{~s}$, which puts an upper limit on the characteristic time for equilibrium. For the $32 A \mathrm{MeV}$ Xe+Sn systems SMF finds isospin equilibrium for impact parameters smaller than 5-6 fm, corresponding to a reaction time of 6 $7 \times 10^{-22} \mathrm{~s}$. For $\mathrm{b}=8 \mathrm{fm}$, isospin equilibrium is not reached for a reaction time of $5 \times 10^{-22} \mathrm{~s}$. Thus the isospin equilibration time for $\mathrm{Xe}+\mathrm{Sn}$ at $32 \mathrm{AMeV}$ can be estimated within 5 and $7 \times 10^{-22} \mathrm{~s}$. The order of magnitude of this time is therefore similar for the two studied systems at Fermi energies. It is around 4 times larger than the one obtained near the interaction barrier.

\subsection{Conclusions on isospin transport}

To summarize we have studied isospin transport for several systems, using different sorting variables and isospin sensitive observables. We compared all data with the results of the same stochastic mean field model. In all cases the agreement between experimental and simulated results is better if the symmetry term of the mean field is asy-stiff, with a potential part linearly increasing with density. We also found that isospin equilibrium is reached for available energies around 8-9 $A \mathrm{MeV}$, independently of the mass asymmetry of the entrance channel. This is also supported by the SMF simulations.

If we compare with other published data, the preference for a potential symmetry energy linearly increasing with density is also supported by time evolution of the isospin of neck fragments [41, compared with a slightly different SMF. And also by the results from the competition between dissipative mechanisms for $\mathrm{Ca}$ induced reactions on $\mathrm{Ca}$ and $\mathrm{Ti}$ at $25 \mathrm{AMeV}$ [2, compared with a CoMD simulation. Results from $\mathrm{Sn}+\mathrm{Sn}$ at 35 and $50 \mathrm{AMeV}$ presented in reference [4]. (several isospin dependent variables), compared to an improved quantum molecular dynamics model, ImQMD, plead for an asy-softer EOS. All these results fit however in the large limits defined in the $L-S_{0}$ plane, see for instance figure 2 of ref. 44.

Ref [45] states that there is no isospin equilibrium for $\mathrm{Sn}+\mathrm{Sn}$ at $35 \mathrm{AMeV}$, in contradiction with our results for the close system $\mathrm{Xe}+\mathrm{Sn}$ at $32 A \mathrm{MeV}$. Thus considering the state of the art on this subject, we have to recognize that extracting the asy-stiffness of the nuclear EOS from isospin diffusion is still an issue.

\section{Isospin effects and energy dissipation}

Nuclear stopping observed in nuclear collisions can be used as a probe for transport properties of nuclear matter [4]. Studies of transport phenomena are of primary importance for understanding the fundamental properties of nuclear matter such as energy dissipation, in-medium nucleon-nucleon cross sections $\left(\sigma_{n n / p p}\right.$ and $\left.\sigma_{n p}\right)$ and related mean free paths or isospin diffusion [47, and refs. therein]. They are critical in the description of the supernova collapse and the formation of a neutron star 48. Transport properties of nuclear matter are also one of the basic ingredients for microscopic models 1, 49, and refs. therein]. In this section, we will present some results concerning the degree of stopping achieved in the most central collisions including, in particular, various projectile-target isospin combinations for the $\mathrm{Xe}+\mathrm{Sn}$ system. We use the large dataset provided by the INDRA collaboration concerning symmetric systems, with total mass between 70 and 400, and incident energy covering the full range of the Fermi energy domain between 10 and $100 \mathrm{AMeV}$.

\subsection{Nuclear stopping observable}

Since we are here interested in the maximal values of stopping reached in nuclear collisions, we select the most central collisions, i.e. collisions corresponding to the largest overlap between the two incoming nuclei. The selection is here done using the total multiplicity of charged products $N_{c h}$ (see sect. A.1). To measure the degree of stopping we use the energy isotropy ratio $R_{E}$ defined on an event-byevent basis as :

$$
R_{E}=\frac{1}{2} \frac{\sum_{i} E_{i}^{\perp}}{\sum_{i} E_{i}^{/ /}}
$$

where the index $i$ runs over the total number of charged particles in the event, $E_{i}$ is the centre-of-mass kinetic energy for particle $i$, the indexes $\perp$ and // stand for transverse and parallel components of $E_{i}$ relative to the beam axis. By construction, this ratio $R_{E}$ takes values between 0 and 1 . Figure 7 displays (a) the total multiplicity distribution $N_{c h}$ and (b) the correlation between the isotropy ratio $R_{E}$ and $N_{c h}$. We can see that $\left\langle R_{E}\right\rangle$ (depicted by the black lines in fig. $7 \mathrm{~b}$ and $\mathrm{d}$ ) reaches an asymptotic value (here close to 0.6 ) at large $N_{c h}$ values. This allows to define a limit at $N_{c h}=38$, indicated by the red line for the correlation in Fig. $7 \mathrm{~b}$ and the red histogram in the $N_{c h}$ distribution in Fig. $7 \mathrm{~A}$, above which the isotropy ratio remains almost constant. The normalized correlation between $R_{E}$ and $N_{c h}$ is also displayed onto Fig. $7 \mathrm{~d}$. The normalization is done on the $z$-axis, by flattening the $N_{c h}$ distribution to the same number of entries; this procedure allows to compare with the same level of statistics all values of $N_{c h}$ multiplicities. We see on the normalized 

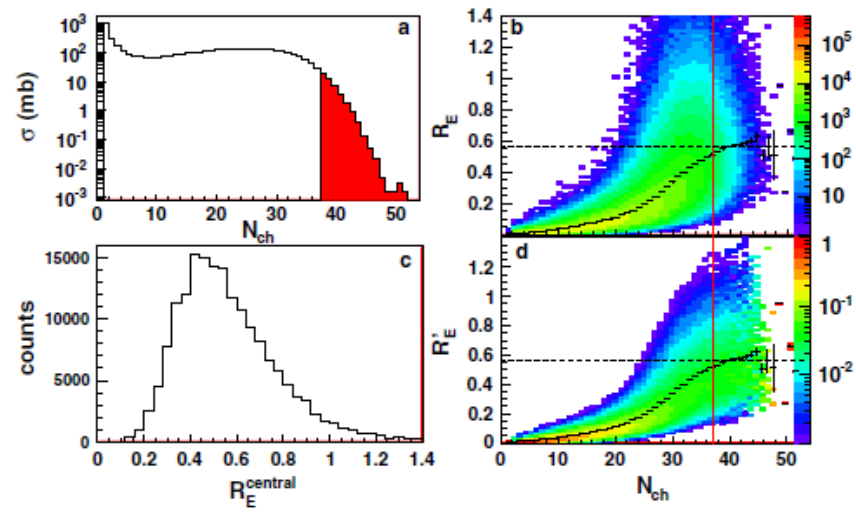

Fig. 7. (Colour on line) Total multiplicity for charged particles $N_{c h}$ (a), correlation between $R_{E}$ and $N_{c} h$ (b), distribution of $R_{E}$ corresponding to the red selection (c), and normalized correlation between $R_{E}$ and $N_{c h}$ (d). From [46].

correlation in Fig. $7 \mathrm{~d}$ that the high values of $R_{E}\left(R_{E}>1\right)$ are significantly reduced, which could indicate that these large $R_{E}$ values are mainly due to the statistics. Fig. 7 . gives the resulting $R_{E}$ distribution given by the red selection on $N_{c h}$. From this distribution, we can extract the mean and standard deviation values of $R_{E}$. To conclude on this point, we have estimated the retained cross section by the total multiplicity selection; this corresponds to roughly $50-100 \mathrm{mb}$, so to an impact parameter range between 0 and $1-1.5 \mathrm{fm}$, whatever the system is.

\subsection{Stopping for central collisions}

We have applied the same procedure for all symmetric systems available in the INDRA collaboration datasets. The mean and standard deviation values of the selected $R_{E}$ distributions are presented in Fig. 8. We notice the evolution of $R_{E}$, from almost 1 for low incident energy to values in between the two limits represented by the dashed curves labeled "entrance channel" and "full stopping". They indicate the $R_{E}$ values for a full stopping $\left(R_{E}=1\right)$ and for no stopping at all; in this latter case, we can simply estimate the expectation values from a simulation of two Fermi spheres in momentum space separated by the relative momentum corresponding to the entrance channel. Hence, this lower limit represents the minimal value of $R_{E}$ in absence of any stopping. We clearly observe two regimes for the stopping; the first one corresponds to low incident energy up to $30-35 \mathrm{AMeV}$. The decreasing stopping is attributed to the progressive disappearance of the nuclear (Mean-Field) effects ruled by 1-Body dissipation [46]. The second regime corresponds to the high incident energy range, from the Fermi energy up to the highest available energy $E_{i n c}=100 \mathrm{AMeV}$. The isotropy ratio remains rather flat, with a clear mass hierarchy : the larger the system, the higher $R_{E}$ is. This phenomenon is attributed to the appareance of the nucleonic degrees of freedom and 2-Body dissipation induced by (elastic) nucleon-nucleon collisions 46. Indeed, these latter become more and more

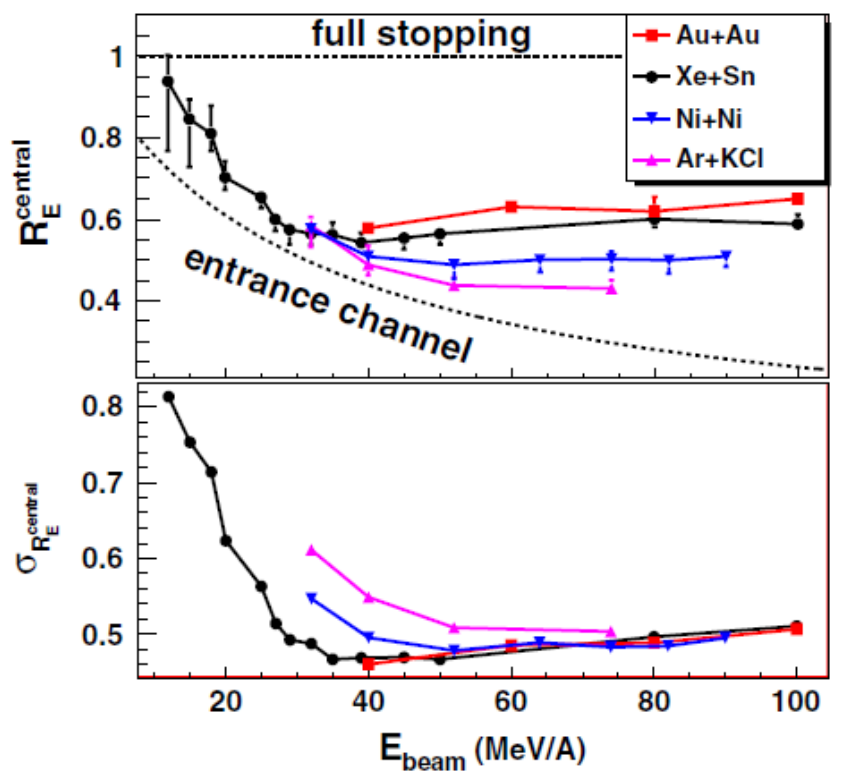

Fig. 8. (Colour on line) Isotropy ratio $R_{E}$ (top: mean values and bottom: rms) as a function of incident energy for various symmetric systems from central collisions. From [46].

likely as we move away from the Fermi energy and, in a Glauber picture, become more and more abundant as the number of participants increases.

\subsection{Isospin effect on nuclear stopping}

We have seen previously that the isotropy ratio can be used as a probe to the energy dissipation/stopping achieved in central collisions. In this section, we give a special emphasis of the eventual isospin effect on the isotropy ratio. To do so, we use various isospin combinations of INDRA Xe+Sn systems: ${ }^{124,129,136} \mathrm{Xe},{ }^{112, \text { nat }}=119,124 \mathrm{Sn}$ at different incident energies: 32,45 and $100 \mathrm{AMeV}$. The same event selection (central collisions selected from $N_{c h}$ ) has been used. We display the results for the corresponding isotropy ratio in table 1 .

We show that, except for the ${ }^{136}$ Xe induced collisions at $E_{\text {inc }} / A=32 \mathrm{MeV}$, all values are statistically compatible at a given incident energy. This seems to indicate that the isospin content does not influence the isotropy ratio and hence the energy dissipation in the Fermi energy range up to a $\delta=(N-Z) / A$ close to $20 \%$. This result is quite surprising since we expect some variations due to the differences on the magnitude of the isovector channels for the nucleon-nucleon cross section $\sigma_{n n / p p}$ and $\sigma_{n p}$ [50 52].

\subsection{Conclusions}

To conclude on these aspects related to the transport properties of nuclear matter, we believe that stopping studies in central collisions can provide new information about 


\begin{tabular}{|c|c|c|c|c|}
\hline System & $\delta=\frac{\left(N_{\text {tot }}-Z_{\text {tot }}\right)}{A_{\text {tot }}}$ & $32 \mathrm{AMeV}$ & $45 \mathrm{AMeV}$ & $100 \mathrm{AMeV}$ \\
\hline${ }^{124} \mathrm{Xe}+{ }^{112} S n$ & 0.119 & $0.54 \pm 0.04$ & $0.53 \pm 0.04$ & $0.58 \pm 0.05$ \\
${ }^{129} \mathrm{Xe}+{ }^{112} \mathrm{Sn}$ & 0.137 & - & - & $0.60 \pm 0.05$ \\
${ }^{124} \mathrm{Xe}+{ }^{124} \mathrm{Sn}$ & 0.161 & $0.54 \pm 0.04$ & - & $0.56 \pm 0.04$ \\
${ }^{129} \mathrm{Xe}+{ }^{\text {nat }} \mathrm{Sn}$ & 0.161 & $0.55 \pm 0.03$ & $0.53 \pm 0.04$ & - \\
${ }^{136} \mathrm{Xe}+{ }^{112} \mathrm{Sn}$ & 0.161 & $0.50 \pm 0.03$ & $0.54 \pm 0.04$ & - \\
${ }^{129} \mathrm{Xe}+{ }^{124} \mathrm{Sn}$ & 0.178 & - & - & $0.59 \pm 0.05$ \\
${ }^{136} \mathrm{Xe}+{ }^{124} \mathrm{Sn}$ & 0.200 & $0.49 \pm 0.03$ & $0.52 \pm 0.05$ & - \\
\hline
\end{tabular}

Table 1. Mean values and standard deviations for $R_{E}$ in central collisions.

the fundamental in-medium quantities such as the nucleonnucleon mean free path, the nucleon-nucleon cross section for example [53]. Moreover, isovector properties of the nuclear interaction like the (reduced) mass splitting between protons and neutrons in the medium can be probed by using asymmetric nuclear matter systems produced with next-generation radioactive beam facilities and detection arrays. This could help to assess from a microscopical point of view the link between the isovector properties of the nuclear interaction and the potential part of the symmetry energy in the nuclear equation of state. As a perspective, all these results concerning the study of stopping in nuclear collisions speak in favour of more detailed analyses both on experimental and theoretical side.

\section{Studies in progress}

\subsection{Symmetry energy, level density parameter and fission barriers}

Technical advances both in producing exotic beams and in detection devices allow to progress in the study of the de-excitation of hot nuclei formed by heavy-ion induced fusion reactions. SPIRAL at GANIL now accelerates exotic Ar beams up to $15 \mathrm{AMeV}$ and $\mathrm{Kr}$ beams around 5-6 $A \mathrm{MeV}$. The $4 \pi$ array INDRA has proven to be an efficient tool to measure simultaneously light charged particles, fission fragments and evaporation residues at low bombarding energy, and was used to scan the de-excitation of medium-mass hot nuclei. Its drawback is the lack of mass identification for the evaporation residues. We thus realized a very ambitious experiment aiming at completely identifying the residue and all emitted particles by coupling INDRA with the large acceptance VAMOS spectrometer 54 56.

Understanding the de-excitation of hot nuclei requires the knowledge of fundamental nuclear parameters, such as level density parameters and fission barriers, to describe the thermal and collective properties that rule the competition between the different decay modes. The isospin content of the compound nucleus has a strong influence on these quantities. The fission barriers strongly depend on the symmetry energy, weakly constrained by the experimental data [57]. The level density parameter, $a$, is related to the effective mass, a property of the nuclear interaction that is sensitive to the neutron and proton content of the nuclei. The effective nucleon mass is expected to decrease with increasing $\mathrm{T}$ while $\mathrm{T} \leq 2 \mathrm{MeV}$. This implies a decrease of the level density parameter but also an increase with $\mathrm{T}$ of the kinetic symmetry energy contribution to the nuclear binding energy $E_{\text {sym }}(T)=b_{\text {sym }}(T) \times(N-Z)^{2} / A$. These effects would experimentally appear as a change in the particle multiplicity and in the relative yields of the exit channels [58. Experimentally $a$ can not be directly measured at high energy, but the temperature $T$ and $1 / T=\mathrm{d} \ln \rho / \mathrm{d} E^{*}$ can be extracted from the exponential slope of kinetic energy spectra of evaporated particles. Multichance emission could be taken into account through statistical model calculations like GEMINI [59. Comparisons with calculations 60] constrain the dependence of $a$ with $E^{*}$ and $T$ and verify the consistency with other data for known isotopes. $a$ was shown to evolve from $\mathrm{A} / 8.5 \mathrm{MeV}^{-1}$ at low temperature to $\mathrm{A} / 15$ $\mathrm{MeV}^{-1}$ around $\mathrm{T}=4-5 \mathrm{MeV}$ 61]. The predicted isospin dependence of level density within the Fermi gas model is a small decrease with increasing (N-Z). A significantly larger dependence would have important implications for other fields (r-process for instance). Different extrapolations starting from stable nuclei have been proposed 62 that lead to quite important variations on the estimated values of the level density parameter. It may even vanish when approaching the proton-drip line. Experimental data far from the valley of stability are very scarce. For that reason, and also because in the multifragmentation process observed at Fermi energies, excited neutron deficient fragments are assumed to be formed, and their de-excitation is not well constrained 22], it is important to get new information on the de-excitation over a large range of isotopes of compound nuclei.

\subsubsection{De-excitation of Ba nuclei formed at low bombarding energy}

Fission barriers strongly depend on the symmetry energy parameter used for calculating the macroscopic part of binding energies. This term is insufficiently constrained by experimental data on the fission barriers [57] of nuclei with $\mathrm{A}=100-180$, where nuclei formed in this intermediate-mass region are able to sustain extreme stresses, including high temperatures, large rotation and deformation.

In this line an experiment was performed with INDRA to study the ${ }^{78,82} \mathrm{Kr}+{ }^{40} \mathrm{Ca}$ reactions at $5.5 \mathrm{AMeV} 63$. At this low incident energy, around the Coulomb barrier, medium-mass compound nuclei are formed in a controlled way in terms of excitation energy and angular momentum. 
The cross section, kinetic energy distribution, angular distribution of fragments with atomic number $3 \leq Z \leq$ 28 and coincidences between light charged particles and fragments were measured. Global features indicate a high degree of relaxation and are compatible with a binary fission from compound nuclei. Inclusive cross-section distri-

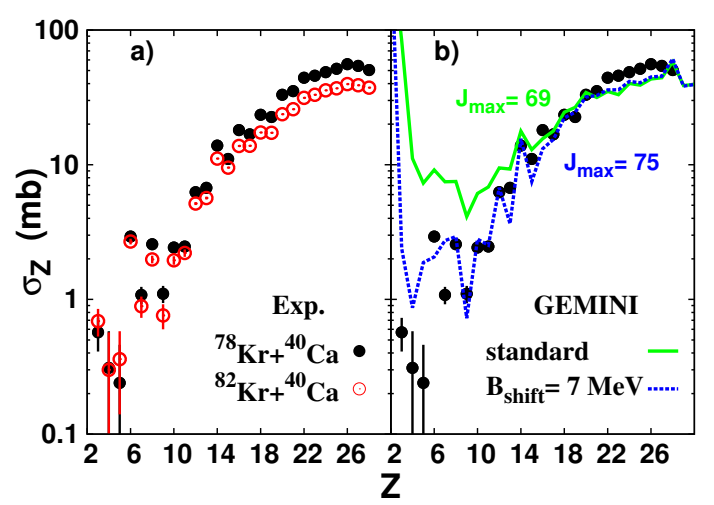

Fig. 9. (Colour on line) Experimental cross-sections for fragments measured in the ${ }^{78} \mathrm{Kr}+{ }^{40} \mathrm{Ca}$ (full circles) and ${ }^{82} \mathrm{Kr}+{ }^{40} \mathrm{Ca}$ (open circles) reactions at 5.5 $\mathrm{AMeV}$. Curves are the predictions of two calculations performed in the framework of the transition state model 64 assuming the fission barriers given by the FRLDM [57] (solid line) and by the FRLDM barriers increased by a constant $\mathrm{B}_{\text {shift }}=7 \mathrm{MeV}$ (dashed line). A level density parameter $a=A / 7 \mathrm{MeV}^{-1}$ is taken for both calculations.

butions of fragments with charge $3 \leq Z \leq 28$, displayed in fig. 9 a, are typical of evaporation plus fission distributions, and a strong odd-even staggering (oes) is observed for $3 \leq Z \leq 12$, indicating that structure effects persist. No isospin dependence of the oes magnitude is clearly visible. The fission channel is $25 \%$ higher for the system with the lowest neutron-to-proton ratio while the evaporation residue cross-sections for both systems are similar within the error bars.

Coincidence measurements between light charged particles and fragments suggest that the light partners in very asymmetric fission are emitted at excitation energies below the particle emission thresholds for both systems: the odd-even staggering of the light fragments cross-sections is not predominantly related to the secondary emission of light particles from excited fragments. The $\sigma_{Z}$ of the light fragments reflects directly the primary fragmentation and thus they provide important constraints on the energetic balance between both fragments.

The $Z$-dependence of the mean value of the total kinetic energy (TKE) indicates dominance of the Coulomb interaction between partners. The width of the TKE distributions signals large fluctuations of the TKE and the ratio $\sigma(T K E) /\langle T K E\rangle$ is roughly constant. The global features of the kinetic energy distributions are not reproduced assuming a total kinetic energy including both contribution extracted from the Viola systematics [65] and from the relative motion.
Statistical calculations assuming spherical fission fragments and finite-range liquid drop fission barriers are not able to explain the experimental features. A fitting procedure assuming a $T K E$ fluctuation given by an average value of the experimental one and a constant shift of $7 \mathrm{MeV}$ on the Finite Range Liquid Drop Model (FRLDM) fission barriers [57] allows to explain both the $Z$-distribution over the whole range of charge-asymmetry and the $Z$ dependence of the $\langle T K E\rangle$ except for $Z \geq 20$, see fig. $9 \mathrm{~b}$. In a recent work [66], the increase of the fission barriers by such a shift was also proposed to reproduce charge distributions in similar reactions at lower angular momenta.

The TKE fluctuation could be related to deformations of the partners indicating the strong influence of the shape parameterization of the potential energy surface in describing the fission process of intermediate mass compound nuclei.

\subsubsection{Level density parameter of Pd nuclei from stability to the proton-drip line}

We studied the de-excitation properties of Pd nuclei formed in collisions between different Ar projectiles and Ni targets: ${ }^{34} \mathrm{Ar}+{ }^{58} \mathrm{Ni},{ }^{36} \mathrm{Ar}+{ }^{58} \mathrm{Ni},{ }^{36} \mathrm{Ar}+{ }^{60} \mathrm{Ni},{ }^{40} \mathrm{Ar}+{ }^{60} \mathrm{Ni}$ and ${ }^{40} \mathrm{Ar}+{ }^{64} \mathrm{Ni}$, at incident energies around $13 \mathrm{~A} . \mathrm{MeV}$, using VAMOS coupled with INDRA. This energy was a compromise between not too large preequilibrium effects and sufficient recoil energy for nuclear charge identification of residues. The exact incident energy for each beam was chosen to get the same excitation energy per nucleon of compound nuclei $(2.9 \mathrm{MeV})$ with very similar angular momentum ranges.

The unstable ${ }^{34} \mathrm{Ar}$ beam was extremely important since it allowed to touch the p-drip line in forming ${ }^{92} \mathrm{Pd}$ : depending on model the drip-line is predicted to be between masses 84 and 89 67. In this case special de-excitation properties might be observed. With the stable ${ }^{36} \mathrm{Ar}$ beam coupled to the ${ }^{60} \mathrm{Ni}$ target the neutron-magic nucleus ${ }^{96} \mathrm{Pd}$ is made.

The detection of complete events should put an additional strong constraint on the values of $a$ for nuclei along the de-excitation chain, provided by the correct weighing of the different exit channels; this was never measured up to now. As a simple example, in the experiment the $\mathrm{Ni}(\mathrm{Ar}, \alpha \times \mathrm{xn}) \mathrm{Ru}$ channel can be distinguished from the $\operatorname{Ni}(\operatorname{Ar}, 2 p(x+2) n) R u$ and $\operatorname{Ni}(\operatorname{Ar}, p d(x+1) n) R u$ channels and correctly weighed. All decay chains can be precisely characterized (isotopic composition of emitted particles and their multiplicity as well as the residue characteristics $(\mathrm{A}, \mathrm{Z})$ and their kinetic energies event by event) and we will obtain the percentage with which different chains lead to the same residue. Note that if the total detected charge is that of $\mathrm{Pd}$, the neutron multiplicity is simply derived by the difference between the compound nucleus mass and those of all detected de-excitation charged products.

Some preliminary data were published, which made use of the INDRA response alone, without identification or calibration 68, 69. The fusion-evaporation cross-section 
seems to decrease by a factor $\sim 2$ for the system close to the drip-line whereas it is constant for the four other systems. INDRA is now fully calibrated, and the complete reconstruction of residue properties in VAMOS is almost achieved. Concerning cross sections we will firstly refine this preliminary analysis, with the full residue angular distributions (no measurements below $7^{\circ}$ were performed with INDRA alone) and secondly determine the process responsible of the missing cross-section. For similar systems (far from the drip-line) fusion-evaporation and fusion-fission cross-sections were found nearly equal 70 , 71. A possibility is thus an increase of the fission crosssection due to a larger fissility parameter $Z^{2} / A$. But as stated above, we might expect exotic processes when nuclei very close to the drip-line are formed. Moreover the compound nuclei excitation energies are close to the multifragmentation threshold. In a Lattice Gas framework, it was shown that both isospin and Coulomb effects lower the transition temperature of the liquid-gas phase transition 72 . Statistical model simulations (GEMINI) will be used to determine the values of $a$, at least for the compound nuclei, including the strong constraints given by the de-excitation processes cross-sections and the weighted deexcitation chains.

\subsection{Symmetry energy and isotopic distributions}

Another observable predicted to be sensitive to the symmetry energy is the shape of isotopic distributions. Such distributions were studied in transport code simulations, AMD (Antisymmetrized Molecular Dynamics) 73 and SMF 23, through the determination of the free energy of fragments in the model connected with their statistical properties, like isoscaling (see section 6.2).

Recently, in the framework of Stochastic Mean Field calculations, M. Colonna 74 demonstrated that the shape of the isotopic distribution can be related to the symmetry energy term of the EOS. In this context, full SMF simulations in a box for unstable matter allow the fragment formation. The isovector fluctuations were estimated as a function of the local density inside the fragmenting system by looking at the variance of the isovector density in cells having the same density. If the equilibrium is reached the quantity $F=T / \sigma$ (where $T$ is the temperature and $\sigma$ is the isovector variance) coincides with the effective symmetry free energy. This analysis of the isotopic distribution of the fragments should thus probe the local symmetry energy of clusterized systems (which is different from the extraction of the total symmetry energy associated with clusterized low-density matter [74, and refs. therein]).

In ref. [75, and refs. therein] the authors simulate headon nuclear collisions at $35 \mathrm{AMeV}$; they construct a global isotopic distribution, $\mathrm{K}(\mathrm{N}, \mathrm{Z})$, by combining together all the yields of fragments (N,Z) obtained in four reaction systems: ${ }^{40} \mathrm{Ca}+{ }^{40} \mathrm{Ca},{ }^{48} \mathrm{Ca}+{ }^{48} \mathrm{Ca},{ }^{60} \mathrm{Ca}+{ }^{60} \mathrm{Ca}$, and ${ }^{46} \mathrm{Fe}+{ }^{46} \mathrm{Fe}$, in order to get a broad range of isotopes. The $\mathrm{K}(\mathrm{N}, \mathrm{Z})$ distributions for each Z-value, were well fitted by a quadratic function

$$
K(N, Z)=\eta(Z)+\xi(Z) N+\zeta(Z) \frac{(N-Z)^{2}}{N+Z}
$$

where $\eta(Z), \xi(Z)$ and $\zeta(Z)$ are the fitting parameters. The obtained parameter of the quadratic term in $(\mathrm{N}-\mathrm{Z})$ is associated by the authors to the symmetry energy coefficient $c_{\text {sym }}(A)$ in the EOS through the relation $\zeta(Z)=$ $c_{\text {sym }}(A) / T$, where $\mathrm{T}$ is the temperature of the system. The symmetry coefficient $c_{\text {sym }}(A)$ is here the sum of a volume and a surface term, $c_{\text {sym }}(A)=c_{\text {sym }}^{v}+c_{\text {sym }}^{s} A^{-1 / 3}$, as in advanced mass formulae [76. The obtained values of $\zeta(Z)$ for each $Z$ have almost no dependence on the charge $\mathrm{Z}(\mathrm{Z}>4)$ (see Fig.3 of Ref. [75). The AMD predictions of constant $\zeta(Z)$ values with increasing $\mathrm{Z}$ indicate that the contribution of the surface term to the symmetry energy is strongly reduced in multifragmentation events. Based on these findings, the authors of Ref. [75] conclude that, at the low density freeze-out stage, the surface term does not contribute strongly to the symmetry energy. Therefore, the symmetry energy, $c_{\text {sym }}(A)$, at finite temperature and subsaturation densities that one can extract from fragment isotopic distributions corresponds to the volume term of the symmetry energy in infinite nuclear matter. This result requires experimental confirmation.

\subsubsection{Experimental methods}

In view of the above considerations, we performed an experiment at GANIL using the VAMOS spectrometer 5456] coupled to the INDRA array. We studied four reactions ${ }^{40} \mathrm{Ca}+{ }^{40} \mathrm{Ca},{ }^{40} \mathrm{Ca}+{ }^{48} \mathrm{Ca},{ }^{48} \mathrm{Ca}+{ }^{40} \mathrm{Ca}$ and ${ }^{48} \mathrm{Ca}+{ }^{48} \mathrm{Ca}$ at $\mathrm{E} / \mathrm{A}=35 \mathrm{MeV}$. The use of a magnetic spectrometer allows one to measure a very wide range of isotopic distributions with the highest mass resolution. Coupling VAMOS and INDRA permits a good impact parameter determination and the use of calorimetry techniques to estimate excitation energies and temperatures in these reactions. In the case of peripheral collisions we measure with VAMOS the isotope production for PLF's produced by deep inelastic mechanism at low excitation energy (i.e. with velocity close to that of the beam). The extraction of the $\zeta$ parameter for each element can provide information about the surface effects in the symmetry energy at finite temperature and at densities close to the saturation density. The acceptance of the VAMOS spectrometer is large enough to measure also isotopic distributions produced in more central collisions. In these more violent events, higher excitation energies are involved and we expect to approach the multifragmentation regime where a study of the isotopic distributions provides access to $\zeta$ and the symmetry energy at sub-saturation densities.

\subsubsection{Isotopic distributions}

Experimental isotopic distributions of fragments with $Z=$ 10,14, 18 and 20 are presented in Fig. 10 for the four analyzed systems. The distributions span over more than 10 

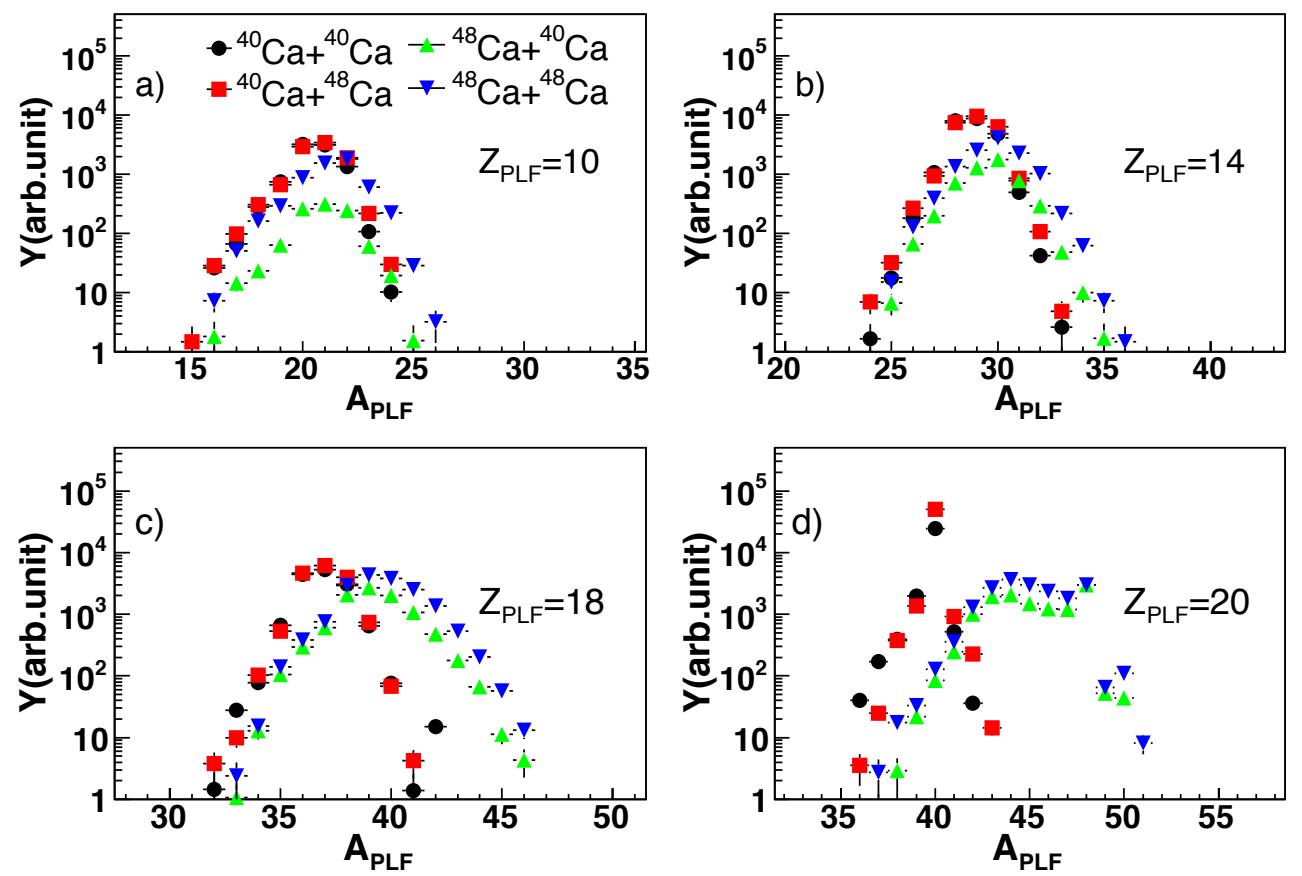

Fig. 10. (Colour on line) Isotopic distributions of fragments with atomic number $Z_{P L F}=10,14,18$ and 20 , produced in the reactions ${ }^{40,48} \mathrm{Ca}+{ }^{40,48} \mathrm{Ca}$ at $E / A=35 \mathrm{MeV}$.

isotopes with relative yields covering 4 orders of magnitude. Very n-rich isotopes are populated reaching a value of $N / Z=1.6$ for $Z_{P L F}=10$ and $N / Z=1.57$ for $Z_{P L F}=$ 18,19 . This ratio exceeds by $11 \%$ the $N / Z$ of the initial neutron-rich projectile $\left({ }^{48} \mathrm{Ca} N / Z=1.4\right)$. We observe the following features:

- an increase of the mean value $\left(\left\langle A_{P L F}\right\rangle\right)$ and width $(\sigma)$ of the distributions as the considered $Z_{P L F}$ increases. For small $Z_{P L F}$ no significant differences in the mean and width of the isotopic distributions for the four systems are observed, while both increase significantly with increasing $Z_{P L F}$ for the n-rich projectile $\left({ }^{48} \mathrm{Ca}\right)$. The effect is most pronounced for $Z_{P L F}=Z_{\text {proj }}$.

- No dependence of the isotopic distributions on the target is observed for the ${ }^{40} \mathrm{Ca}$ projectile, whereas a shift toward higher $\left\langle A_{P L F}\right\rangle$ and $\sigma$ is observed when moving from the ${ }^{40} \mathrm{Ca}$ to ${ }^{48} \mathrm{Ca}$ target.

The shift of the mean values and the broadening of the distributions as the n-richness of the system increases can be understood in terms of available neutrons. The evolution of the behaviour observed as $Z_{P L F}$ increases reflects the evolution of the products with the centrality of the collision, therefore with the interaction time and the flux of nucleons exchange.

The analysis of these results following the lines of refs. [74, 75] is in progress and should provide new information on the symmetry energy term of EOS of nuclear matter.

\subsection{Using a new approach for solving the Boltzmann-Langevin equation}

As we discussed in section 2, for central collisions the theoretical approach SMF which applies rather efficiently at larger bombarding energies (Xe+Sn at $45 \mathrm{AMeV}$ ), does not extend to lower energies. From a microscopic point of view, the drop in fragment multiplicity in proximity of the low-energy multifragmentation threshold may result from a complex interplay between spinodal instabilities, which tend to fragment the system in several portions of comparable size, and the coalescence effect of the mean-field, which tends to revert the system to a compact shape. This picture requires first of all that the many-body description (fragment observables) at low bombarding energies should be done without sacrifying the one-body properties, like the spinodal behaviour and, secondly, that the fermionic model is sufficiently stable (against turning into a Boltzmann statistics), which is a fundamental requirement for following relatively long-time processes like coalescence effects. A suited approach to extend to lower energies was therefore developed with the aim of keeping the same accuracy in describing isospin and transport properties (i.e. the isovector-dependent mean field) along a larger incident-energy interval which includes also the low-energy threshold of multifragmentation. Such extension required the solution of the Boltzmann-Langevin (BL) equation in three dimensions in order to describe the fluctuation phenomenology in full phase space.

A discussion on the problems which may be encountered in introducing a Langevin term in a transport model is presented by Chapelle et al. in ref. [77, and a solution 
was the method introduced by Rizzo et al. in ref. 78, tested in nuclear matter. The recommendation from the above references was to pay special attention to the Pauli blocking; this becomes therefore the direction followed for building the Boltzmann-Langevin One Body model, BLOB [79, 80]. With the BLOB simulations, the multifragmentation process can be followed down to low energy where the system explores instabilities against density fluctuations when low densities are attained, and a characteristic spinodal behaviour stands out. The fact that, in this situation, the amplitude of the unstable modes grows according to the specific dispersion relation associated with the employed mean-field interaction ensures that the Langevin term is implemented satisfactorily.

With the BLOB model head-on collisions for the system ${ }^{136} \mathrm{Xe}+{ }^{124} \mathrm{Sn}$ can be described quantitatively down to low energy, as shown in fig. 11 for four incident energies. At $24 \mathrm{AMeV}$, large fragment multiplicities are present. At $32 \mathrm{AMeV}$, conversely to SMF simulations, one observes a spinodal behaviour. $45 \mathrm{AMeV}$ corresponds with the multifragmentation regime and to the situation when secondary decay does not modify the primary distribution of fragments, and $58 \mathrm{AMeV}$ is at the fading side of the spinodal multifragmentation process.

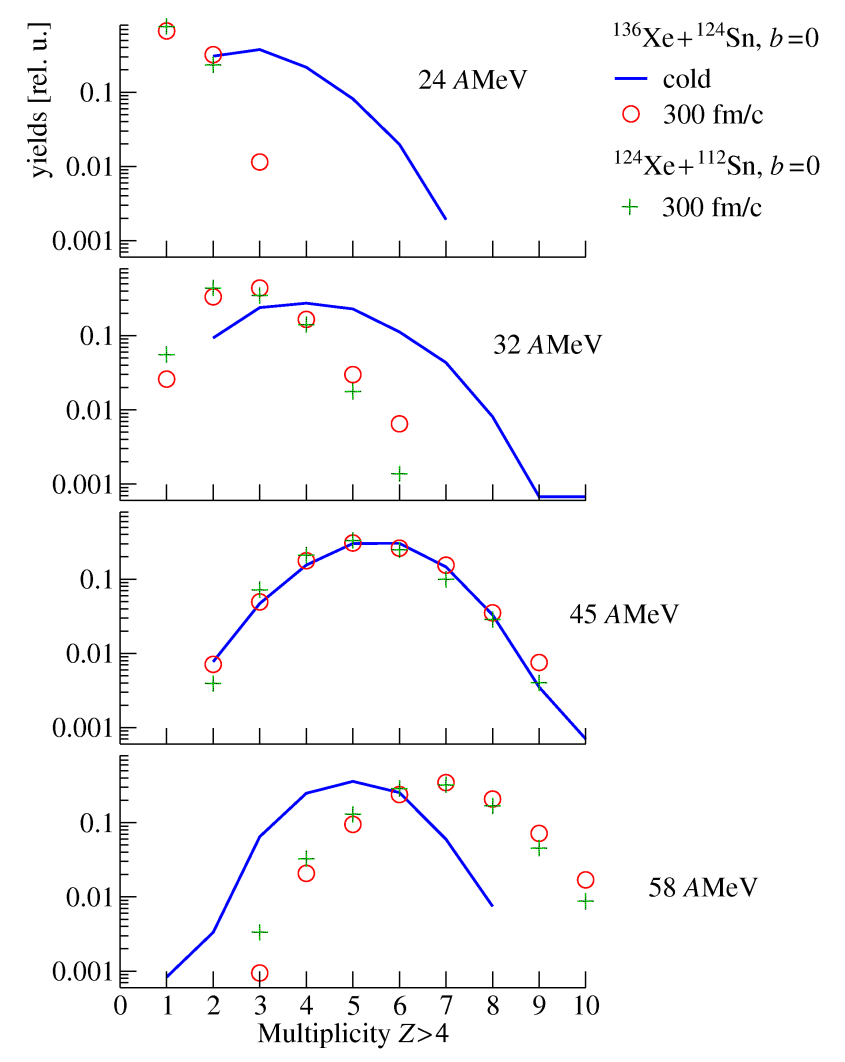

Fig. 11. Multiplicity distribution of fragments with $Z>4$ obtained for four incident energies with BLOB, for ${ }^{136} \mathrm{Xe}+{ }^{124} \mathrm{Sn}$ and ${ }^{124} \mathrm{Xe}+{ }^{112} \mathrm{Sn}$ reactions, at $300 \mathrm{fm} / \mathrm{c}$ for $b=0$. The first system is also studied after secondary decay.
To compare more quantitatively with the data discussed in section 2, we selected collisions with impact parameters smaller than $4 \mathrm{fm}$ from simulated events (asystiff EOS) for the system ${ }^{136} \mathrm{Xe}+{ }^{124} \mathrm{Sn}$ at $32 \mathrm{AMeV}$. After de-excitation of the hot fragments one finds $\left\langle M_{\text {frag }}\right\rangle=4.5$, $\left\langle Z_{l c p}\right\rangle=36.0,\left\langle Z_{b 5}\right\rangle=60.6$. These preliminary low statistics results can be compared to the experimental values $\left\langle M_{\text {frag }}\right\rangle=5.5,\left\langle Z_{l c p}\right\rangle=26.7,\left\langle Z_{b 5}\right\rangle=71.0$. While underestimation of the fragment multiplicity and of $Z_{b 5}$ are of the same order of magnitude as that observed at $45 \mathrm{AMeV}$ with SMF, the value of $Z_{l c p}$ is in better agreement with the data, because preequilibrium emission is reduced.

Thus the BLOB model extends the description of multifragmentation to lower energies than the SMF model. It is due to the following mechanism: fluctuations have the effect of reducing the fraction of energy spent in the emission of light-particles (the so-called preequilibrium). More energy is therefore available for feeding the development of inhomogeneities and, at the same time, for imparting a large kinetic energy to them: the dynamics results more explosive and is able to drive the fragment separation and to produce events of large fragment multiplicity. Such modelling approach results then in a more correct description of the velocity profile of fragments. It should allow to get information on the symmetry energy stiffness by looking, for instance, at the evolution of the average $\mathrm{N} / \mathrm{Z}$ of light fragments vs their centre of mass kinetic energy, as proposed in [81, 82].

\section{Foreseen studies}

The advent of radioactive intense beam facilities altogether with the development of new-generation $4 \pi$ arrays 83 will allow to deeply investigate the physics of isospin in the forthcoming years. Indeed, a large panoply of n-rich and p-rich beams will become available with bombarding energies from the Coulomb barrier up to few hundreds of $\mathrm{MeV}$ per nucleon. From a perspective point of view [84, 85], the exploration of the isospin degree of freedom through heavy-ion induced reactions will bring up the possibility of studying :

- limiting temperatures in hot N/Z asymmetric nuclear systems: Coulomb versus isovector instabilities,

- isospin dependence of nuclear level densities in warm nuclei: continuation of INDRA-VAMOS program (section 5.1.2),

- isospin diffusion and migration through dissipative collisions,

- isospin dependence of the nuclear phase diagram: phase transitions and coexistence lines, spinodal (mechanical) versus isovector (chemical) instabilities, hot versus "cold" multifragmentation,

- the nuclear symmetry energy directly from fragment isotopic distributions: isoscaling as detailed below.

In the mean time, coupling the FAZIA demonstrator with INDRA, we foresee the following investigation. We performed theoretical study in the framework of a $3 D$ Lattice-Gas Model, (LGM) 72. In this simulation, we 
have implemented an isocalar + isovector + Coulomb Hamiltonian in order to evaluate the combined effects of nuclear, isospin and Coulomb interactions concerning the fragment production of excited nuclear systems produced in heavyion reactions in the Fermi energy domain. Details concerning the implementation and results from this model can be found in 72 , 86.

\subsection{Density functional}

To relate the system properties to a macroscopic description in term of density functional, we have implemented a Liquid Drop parametrization for the internal energy with coefficients which are density-dependent. Thus, the internal energy $E_{\text {int }}$ of the nuclear system at density $\rho$ and isospin content $\delta=(N-Z) / A$ can be expressed as:

$$
\begin{aligned}
& E_{\text {int }}^{L D}(\rho, \delta)= \\
& \quad\left[a_{v}(\rho)+c_{\text {sym }}^{v}(\rho) \delta^{2}\right] A+\left[a_{s}(\rho)+c_{\text {sym }}^{s}(\rho) \delta^{2}\right] A^{2 / 3} \\
& \quad+\alpha_{c}(\rho) Z^{2}
\end{aligned}
$$

where $a_{v}, c_{\text {sym }}^{v}, c_{\text {sym }}^{s}, a_{s}$ and $\alpha_{c}$ are respectively the volume, symmetry (volume and surface terms), surface and Coulomb coefficients. The label $L D$ stands here for Liquid Drop.

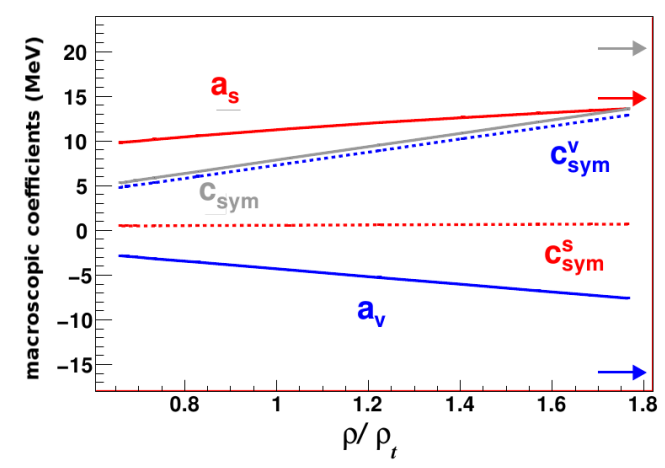

Fig. 12. Density dependence of the $L D$ coefficients. The arrows indicate the values obtained for $c_{s y m}^{v} a_{s}$ and $a_{v}$ at saturation density/zero temperature. From 87.

Fig. 12 shows the density dependence of the $L D$ coefficients. We notice that all coefficients are decreasing with the density and that the surface term of the symmetry energy $c_{\text {sym }}^{s}$ is here equal to zero; the main contribution for the symmetry term is indeed the volume coefficient $c_{\text {sym }}^{v}$ in this implementation of $L G M$ [72], as also found in AMD simulations (see section 5.2).

\subsection{Isospin dependence of the symmetry energy}

Isotopic scaling has been experimentally observed several years ago by the $M S U$ group [88, 89], and also found later in various experimental data $90-92$. It is built upon the ratio of production yields obtained from two sets of nuclear reactions having the same total mass $A$ but different isospin ratios $N / Z$ and $N^{\prime} / Z^{\prime}$ (which are hereafter labeled (1) and (2)). More precisely, this is an isotopic scaling law, abbreviated as isocaling, which expresses the ratio $R_{21}(Z, N)$ between the production yields $Y_{i}(Z, N)$, for both reactions, of a fragment defined by $Z$ protons and $N$ neutrons as :

$$
R_{21}(Z, N)=\frac{Y_{2}(Z, N)}{Y_{1}(Z, N)} \propto \exp (\beta Z+\alpha N)
$$

where $\alpha$ and $\beta$ are the two isoscaling parameters related respectively to the number of neutrons $N$ and the number of protons $Z$. This scaling is characterized by parallel straight lines in a logarithmic plot of $R_{Z, N}$ as a function of $N$ or $Z$ whatever is the fragment. The slope of the straight lines is either $\alpha$ or $\beta$ depending on the representation, for instance it is $\alpha$ on Fig. 13 obtained from $L G M$ simulations.

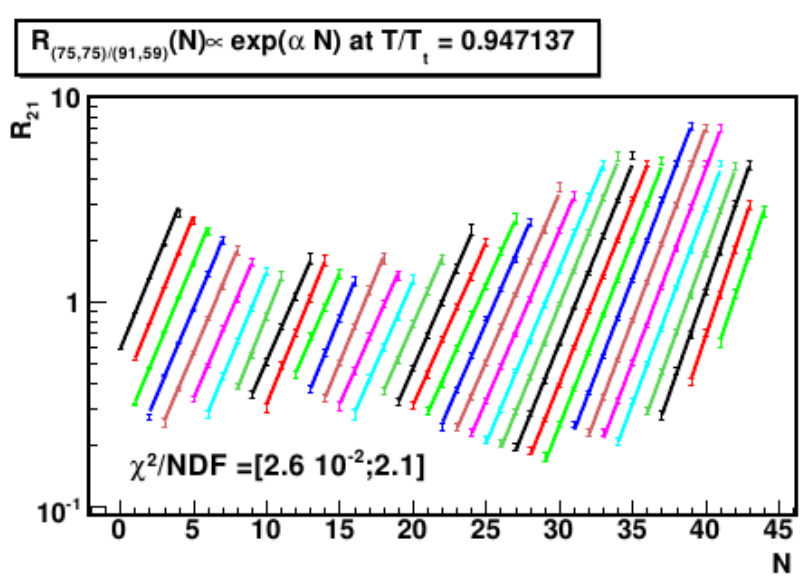

Fig. 13. (Colour on line) Yield ratio $R_{2} 1(Z, N)$ as a function of the number of neutrons $N$ for nuclei with $Z=1-36$ for LGM simulations. From 87.

It has been proposed to relate this experimental evidence for the isocaling to fundamental properties of the nuclear equation of state, namely the symmetry energy and its coefficient $c_{\text {sym }}$ (see Eq. 8). Different parameterizations have been derived either from macroscopic or microscopic approaches.

\subsubsection{Macroscopic approach}

The first parametrization is based on the macroscopic approach from statistical models, in the grand-canonical framework 93. In this specific case, the isocaling parameter $\alpha$ is related to the symmetry energy coefficient $c_{\text {sym }}$ through :

$$
c_{\text {sym }}(Z)=\frac{\alpha(Z) T}{4\left(Z_{2}^{2} / A_{2}^{2}-Z_{1}^{2} / A_{1}^{2}\right)}
$$


where $Z_{1}, A_{1}$ and $Z_{2}, A_{2}$ are the total atomic numbers and masses of the two distinct isospin systems, $T$ is the temperature, and $\alpha(Z)$ is the isocaling coefficient for fragment with charge $Z$. We can see the results of Eq. 10 plotted as dashed curves on Fig. 14. Each curve corresponds to a given $Z$, varying from $Z=2$ to $Z=7$. We restrict here the isoscaling to light fragments as it is usually done in experimental conditions $88,90,92,94$. The values are far away from the true ones displayed by the symbols as it has been already observed in [95]. Several reasons could explain this large discrepancy. Eq. 10 is not exact and has been derived from a macroscopic framework where the many-body correlations are supposed to be exhausted entirely by clusterization [87. This is a rather crude approximation because it appears to be strongly affected by conservation law, combinatorial effects 96] but also by secondary decay effects 97. At last, Eq. 10 is actually related to the symmetry free energy which should correspond to the symmetry energy only when $T \rightarrow 0$.

\subsubsection{Microscopic approach}

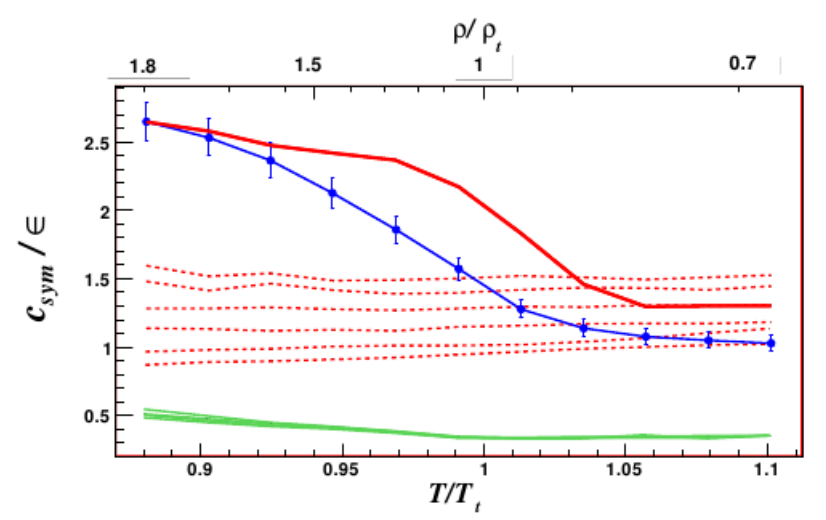

Fig. 14. (Colour on line) Various evaluations of the symmetry energy coefficients (see text) compared to the true one (symbols). Green dashed curves: Eq. 10 red dotted curves: Eq. 11. thick red line is for the largest cluster. From [87.

A second parametrization, derived from the quantal microscopic model $A M D$ in the fragmentation regime [75], has been also established:

$$
c_{\text {sym }}(Z)=\frac{\alpha(Z) T}{4\left(Z^{2} /<A_{1}>^{2}-Z^{2} /<A_{2}>^{2}\right)}
$$

where $\left\langle A_{i}\right\rangle$ is here the average mass number for a fragment with charge $Z$ in the reaction $(i)$. The results are displayed by the dotted light grey (flat) curves in fig 14 for the same range of atomic number $(Z=2-7)$ as previously. We notice that the values are quite correct for the low density/high temperature domain, below (above) the density/temperature transition. This is indeed expected since Eq. 11 has been derived for the fragmentation regime only. For the high density/low temperature domain, the agreement is not anymore observed. This reflects again the difficulty to extract reliable values for $c_{\text {sym }}$ from the isoscaling parameters extracted by looking at light fragments $(Z<8)$ where combinatorial and secondary decay effects may blur the signal.

\subsection{Isoscaling from the largest fragment}

When analysing the results from $L G M$, we notice that the mass distribution includes a large percolating cluster which contains most information on the thermodynamics [72, 98. Therefore, the isotopic distribution of the largest cluster may be more sensitive to the symmetry energy of the fragmenting system. We then apply Eq. 11 to the largest cluster in the event, and this is plotted in Fig. 14 as a continuous thick red line. We observe a better, though still qualitative, agreement with the true value from the model. This is particularly interesting since the largest fragment is by definition bound in LGM [98; this means that this is the final distribution of the largest fragment which is here analyzed, avoiding thus the secondary decay problem pointed out previously. In the vivid perspective of obtaining experimental measurements of isotopic distributions for heavy fragments from experiments coupling recoil spectrometers with large acceptance arrays like MARS with NIMROD at Texas $A \& M$ [99] or VAMOS with INDRA at GANIL [100, or new generation $4 \pi$ arrays like FAZIA [83] associated with radioactive beam facilities, we could expect to get unprecedented results concerning the estimation of the symmetry energy and its associated density/temperature dependence in heavy-ion collisions.

\section{General conclusion}

In this review paper, we gathered all the isospin effects observed in experiments performed with INDRA, essentially with stable beams, which limits the explored $\mathrm{N} / \mathrm{Z}$ range $(1-1.5)$. Nuclear reactions were studied from the barrier to $100 \mathrm{AMeV}$. The Fermi energy domain is well suited for constraining the symmetry energy term of the EOS as the mean field still plays an important role. Subsaturation and moderate suprasaturation densities are explored during these collisions.

For central collisions, we did not observe isospin influence on the stopping between 30 and $100 \mathrm{AMeV}$ in the studied isospin range. On the other hand isospin effects are visible in various properties of the multifragmentation process, but did not allow to constrain the symmetry energy when compared to the SMF transport code. The BLOB model now allows to extend the data-model comparisons to lower energies, which will be done in the near future.

Investigating isospin diffusion as a function of dissipation on a rather large range of impact parameters, we showed that, within the SMF framework, the potential 
part of the symmetry energy linearly increases with density. We also demonstrated that isospin equilibration occurred for semi-central collisions with 8-9 $A \mathrm{MeV}$ available energy. These two properties were observed for both a symmetric and a very asymmetric system.

In a foreseeable future we will couple the FAZIA demonstrator with INDRA. FAZIA should perform isotopic identification for $\mathrm{Z}$ up to 25 . Tke knowledge of the mass of rather heavy fragments on a large solid angle is expected to permit a step forward in constraining the density dependence of the symmetry energy and to give better insight into isospin effects in nuclear reactions.

\section{A Experimental selections with INDRA}

Nucleus-nucleus collisions are classified through their violence, which reflects the impact parameter. INDRA is particularly efficient for central to semiperipheral collisions at intermediate energy. Around and above the Fermi energy most of the collisions end-up with two big nuclei, remnants of projectiles (QP) and target (QT). A fraction of collisions also presents a copious and fast emission of particles and light fragments with velocities intermediate between those of the QP and QT. For central collisions topology selectors allow to isolate quasifusion reactions whereas impact parameter selectors are able to isolate the most central collisions. Depending on collisions and required studies, different selections are used (see for example [14, 22]). As INDRA does not detect neutrons, selections do not lead to $\mathrm{N} / \mathrm{Z}$ reconstructions of nuclear sources.

\section{A.1 Event selection for central collisions}

A two step procedure is used to select central collisions. We shall distinguish, for the second step, quasifusion (QF) sources selections, for which one uses a topology selector (events with isotropic shape in velocity space), from global selections of the most central collisions which need an impact parameter selector. At intermediate energies there are large fluctuations in the exit channel associated to a given impact parameter, as shown in stochastic transport models [23, and such a distinction makes sense.

The first step consists in keeping the events for which a quasi-complete detection of the reaction products has been achieved. Significant fractions: $\geq 77-80 \%$ of the charge of the system, $Z_{\text {sys }}=Z_{\text {proj }}+Z_{\text {targ }}$, is required to be measured for every event. In the second step we need a topology selector in order to select well defined nuclear source issued from quasifusion reactions. The flow angle $\left(\Theta_{\text {flow }}\right)$ selection [14, 101, 102, is largely used. This global variable is defined as the angle between the beam axis and the preferred direction of emitted matter in each event. It is determined by the energy tensor calculated from fragment $(Z \geq 5)$ momenta in the reaction centre of mass. Quasifusion events have no memory of the entrance channel and should be isotropic while binary dissipative collisions are focused at small $\Theta_{\text {flow }}$. Thus, by selecting only large flow angles, fusion events can be well isolated.
The minimum flow angle chosen is $60^{\circ}$ for collisions in the incident energy range 30-50 $\mathrm{AMeV}$. The present selection corresponds to measured cross sections decreasing from 30-40 to 20-25 mb when the incident energy goes from 30 to $50 \mathrm{AMeV}$ for $\mathrm{Xe}+\mathrm{Sn}$ collisions. By taking into account detection efficiency and biases due to the selection (quasi-complete events and flow angle selection) the total cross section for the formation of compact fused systems is estimated to decrease from 200-250 to $80-150 \mathrm{mb}$ between 30 and $50 A \mathrm{MeV}$ [18, 28].

In the experiment leading to the results presented in sections 2 and 3.1 .2 we collected a very large number of events; it thus became possible to perform more severe selections, in order to extrapolate different variables to the values corresponding to a perfect detection of charges, $\mathrm{Z}_{t o t}=104$. We proceed as follows: we considered, for each system, three batches of events with the conditions $\mathrm{Z}_{t o t} \geq 80$, 90 and 95 . We calculated average multiplicities of LCP, $M_{l c p}$ and of fragments with lower limits $\mathrm{Z}=3\left(M_{f 3}\right)$ and $\mathrm{Z}=5\left(M_{\text {frag }}\right)$. We also determined the total charge bound in LCP or fragments, $Z_{l c p}, Z_{b 3}, Z_{b 5}$ respectively. It appeared that all these variables linearly evolve when plotted $v s$ the average values of $\mathrm{Z}_{t o t}$. These linear relations were used to extrapolate the multiplicities and $\mathrm{Z}_{\text {bound }}$ values to what they would be for a perfect detection. The consistency of the procedure was verified by considering the sum $Z_{l c p}+Z_{b 3}$; it was in all cases equal to $104 \pm 0.01$, except for ${ }^{136} \mathrm{Xe}+{ }^{112} \mathrm{Sn}$ at $32 \mathrm{AMeV}$ where it amounts to 104.3 .

More recently another topology selector was proposed to select QF sources. For symmetric systems, taking advantage of the excellent quality detection of INDRA in the centre of mass forward hemisphere, the quantity $V_{\text {bigiso }}$ $=V_{\text {par }}^{2}-0.5 V_{\text {per }}^{2}$ for the heaviest fragment was calculated [37]; $V_{\text {par }}$ and $V_{\text {per }}$ refer to centre of mass velocity components parallel and perpendicular to the beam direction. A value of $V_{\text {bigiso }}$ close to zero is expected for $\mathrm{QF}$ events, for which the velocity distribution of the heaviest fragment must be isotropic. An example of such a selection is shown in figure 15 for different $\mathrm{Xe}+\mathrm{Sn}$ reactions at $32 \mathrm{AMeV}$ incident energy.

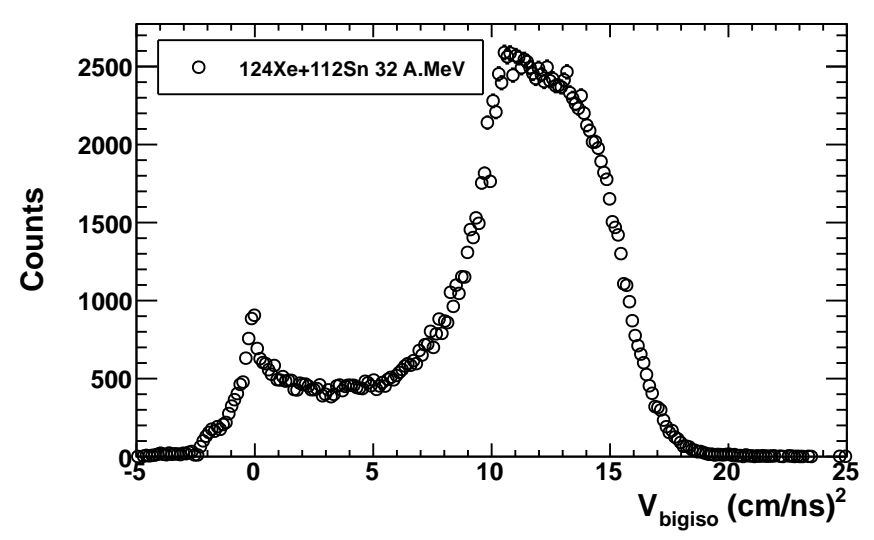

Fig. 15. $V_{\text {bigiso }}$ distributions for a $\mathrm{Xe}+\mathrm{Sn}$ reaction at $32 \mathrm{~A} \mathrm{MeV}$ incident energy. From 37. 
When one wants to select the most central collisions as for stopping studies an impact parameter selector must be preferred. With such a selection mean properties of a well defined class of collisions can be extracted. Since quantities involving both longitudinal and tranverse directions will be used to evaluate the stopping, it is not suitable to use vector variables such as, for instance the transverse energy [35, 103] which could introduce autocorrelations. A scalar variable is desirable an a natural choice is the total multiplicity of detected charged products, $N_{c h}$.

\section{A.2 Selection of quasiprojectiles and impact parameter evaluators}

As previously, a two step selection is applied. A first and simple selection requires that the total detected charge amounts to at least 90 (80)\% of the charge of the projectile (in the forward part of the centre of mass).

A further selection must be done to select the "quasiprojectile". We do not intend to isolate a "source", but rather to select a forward region in phase space where the detected products have a small probability to result from emission by the quasi-target. For symmetric systems, it is done by a cut at the centre-of-mass velocity; we only keep the forward part for which, as previously said, detection is excellent. For asymmetric systems like $\mathrm{Ni}+\mathrm{Au}$, the target being more than three times heavier than the projectile, some particles from the target would be kept. Thus the cut was made at the nucleon-nucleon velocity. The quasi-projectile selection only keeps particles and fragments with a parallel velocity higher than the nucleonnucleon velocity.

So, in the following we call "quasi-projectile" (QP) the ensemble of charged products which have a velocity higher than the centre of mass (nucleon-nucleon for asymmrtric systems) velocity, without prejudice on the equilibration of any degree of freedom of the ensemble so defined.

To study the evolution of observables and to compare with theoretical simulations, impact parameter evaluators have to be used. First of all, as early mentioned, we have chosen the transverse energy of light charged particles (LCP, $\mathrm{Z}=1,2), E_{t r 12}$, but now corresponding to the forward part of the centre of mass. This observable is quite well correlated with the impact parameter, especially for peripheral and semiperipheral collisions, and well suited to the data because the INDRA array is very efficient (85\%) for LCP's.

We have also sorted the events as a function of the dissipated energy, calculated in a binary hypothesis, with the following assumptions:

i) the quasi-projectile velocity, $V_{Q P}^{r e c}$, is equal to the measured velocity of the single fragment, or reconstructed from the velocity of all the fragments it contains,

ii) the relative velocity between the quasi-projectile and the quasi-target is determined as if the collision was purely binary, without mass exchange:

$$
V_{\text {rel }}=V_{Q P}^{r e c} \times \frac{A_{\text {tot }}}{A_{\text {target }}}
$$
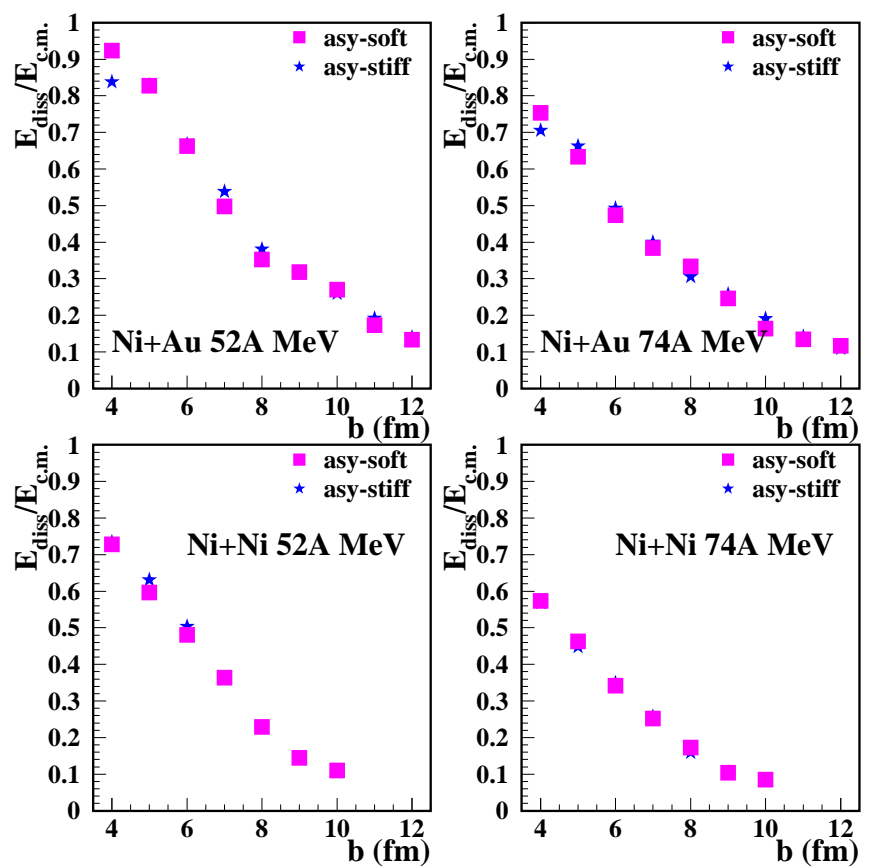

Fig. 16. (Colour on line) Correlation between $E_{\text {diss }} / E_{c m}$ and the true impact parameter for two reactions and two incident energies. Stars (squares) correspond to SMF simulations (see section 2.1 with an asy-stiff (asy-soft) symmetry energy. From [32.

and thus the total dissipated energy reads:

$$
E_{\text {diss }}=E_{\text {c.m. }}-\frac{1}{2} \mu V_{\text {rel }}^{2},
$$

with $\mu$ the initial reduced mass. It is demonstrated in 104 105] that the velocity of the QP is a good parameter for following the dissipated energy, except in very peripheral collisions, due to trigger conditions. Moreover, it is shown in figure 16 that in transport model simulations (SMF, see section 2.1 $E_{\text {diss }}$ gives a good measure of the impact parameter when using the sorting of events as previously defined.

\section{References}

1. C. Fuchs and H. H. Wolter, P. Chomaz, F. Gulminelli, W. Trautmann and S. J. Yennello, eds., Dynamics and Thermodynamics with nuclear degrees of freedom (Springer), vol. 30 of Eur. Phys. J. A, 5-22 (2006).

2. M. Di Toro, V. Baran, M. Colonna, G. Ferini, T. Gaitanos et al., Prog. Part. Nucl. Phys. 62, 389 (2009).

3. V. Baran, M. Colonna, V. Greco and M. Di Toro, Phys. Rep. 410, 335 (2005).

4. J. Rizzo, M. Colonna, V. Baran, M. Di Toro, H. H. Wolter et al., Nucl. Phys. A 806, 79 (2008).

5. P. Napolitani, M. Colonna, F. Gulminelli, E. Galichet, S. Piantelli et al., Phys. Rev. C 81, 044619 (2010). 
6. P. Chomaz, V. Duflot and F. Gulminelli, Phys. Rev. Lett. 85, 3587 (2000); P. Chomaz and F. Gulminelli, Physica A 330, 451 (2003).

7. M. Pichon, B. Tamain, R. Bougault, F. Gulminelli, O. Lopez et al. (INDRA and ALADIN collaborations), Nucl. Phys. A 779, 267 (2006).

8. E. Bonnet, D. Mercier, B. Borderie, F. Gulminelli, M. F. Rivet et al. (INDRA Collaboration), Phys. Rev. Lett. 103, 072701 (2009).

9. C. Ducoin, P. Chomaz and F. Gulminelli, Nucl. Phys. A 781, 407 (2007).

10. J. Pouthas, B. Borderie, R. Dayras, E. Plagnol, M. F. Rivet et al., Nucl. Instr. and Meth. in Phys. Res. A 357, 418 (1995).

11. J. Pouthas, A. Bertaut, B. Borderie, P. Bourgault, B. Cahan et al., Nucl. Instr. and Meth. in Phys. Res. A 369, 222 (1996).

12. P. Chomaz, F. Gulminelli, W. Trautmann and S. J. Yennello, eds., vol. 30 of Eur. Phys. J. A (Springer) (2006).

13. Bao-An Li, Lie-Wen Chen and Che Ming Ko, Phys. Rep. 464, 113 (2008).

14. J. D. Frankland, C. O. Bacri, B. Borderie, M. F. Rivet, M. Squalli et al. (INDRA Collaboration), Nucl. Phys. A 689, 905 (2001).

15. G. Tăbăcaru, B. Borderie, A. Ouatizerga, M. Pârlog, M. F. Rivet et al. (INDRA Collaboration), Nucl. Instr. and Meth. in Phys. Res. A 428, 379 (1999).

16. M. Pârlog, B. Borderie, M. F. Rivet, G. Tăbăcaru, A. Chbihi et al. (INDRA Collaboration), Nucl. Instr. and Meth. in Phys. Res. A 482, 674 (2002).

17. M. Pârlog, B. Borderie, M. F. Rivet, G. Tăbăcaru, A. Chbihi et al. (INDRA Collaboration), Nucl. Instr. and Meth. in Phys. Res. A 482, 693 (2002).

18. F. Gagnon-Moisan, E. Galichet, M. F. Rivet, B. Borderie, M. Colonna et al. (INDRA Collaboration), Phys. Rev. C 86, 044617 (2012).

19. G. J. Kunde, S. Gaff, C. K. Gelbke, T. Glasmacher, M. J. Huang et al., Phys. Rev. Lett. 77, 2897 (1996).

20. M. F. Rivet, C. O. Bacri, B. Borderie, J. D. Frankland, M. Assenard et al. (INDRA Collaboration), Phys. Lett. B 430, 217 (1998).

21. P. Chomaz, M. Colonna and J. Randrup, Phys. Rep. 389, 263 (2004).

22. B. Borderie and M. F. Rivet, Prog. Part. Nucl. Phys. 61, 551 (2008).

23. M. Colonna, M. Di Toro, A. Guarnera, S. Maccarone, M. Zielinska-Pfabé et al., Nucl. Phys. A 642, 449 (1998).

24. M. Colonna, G. Fabbri, M. Di Toro, F. Matera and H. H. Wolter, Nucl. Phys. A 742, 337 (2004).

25. V. Baran, M. Colonna, M. Di Toro, V. Greco, M. Zielinska-Pfabé et al., Nucl. Phys. A 703, 603 (2002).

26. J. D. Frankland, B. Borderie, M. Colonna, M. F. Rivet, C. O. Bacri et al. (INDRA Collaboration), Nucl. Phys. A 689, 940 (2001).

27. D. Durand, Nucl. Phys. A 541, 266 (1992).
28. S. Hudan, A. Chbihi, J. D. Frankland, A. Mignon, A. Botvina et al. (INDRA Collaboration), Phys. Rev. C 67, 064613 (2003).

29. S. Piantelli, B. Borderie, E. Bonnet, N. Le Neindre, A. R. Raduta et al. (INDRA Collaboration), Nucl. Phys. A 809, 111 (2008).

30. V. Baran, M. Colonna, M. Di Toro, M. ZielinskaPfabé and H. H. Wolter, Phys. Rev. C 72, 064620 (2005).

31. E. Galichet, M. F. Rivet, B. Borderie, M. Colonna, R. Bougault et al. (INDRA Collaboration), Phys. Rev. C 79, 064614 (2009).

32. E. Galichet, M. Colonna, B. Borderie and M. F. Rivet, Phys. Rev. C 79, 064615 (2009).

33. E. Galichet, M. F. Rivet, B. Borderie, M. Colonna, R. Bougault et al., Int. J. of Modern Phys. E 19, 1534 (2010).

34. T. Lefort, D. Doré, D. Cussol, Y. Ma, J. Péter et al. (INDRA Collaboration), Nucl. Phys. A 662, 397 (2000).

35. E. Plagnol, J. Łukasik, G. Auger, C. O. Bacri, N. Bellaize et al. (INDRA Collaboration), Phys. Rev. C 61, 014606 (1999).

36. M. Kabtoul, R. Bougault, M. Colonna and E. Galichet (INDRA Collaboration), J. D. Frankland, A. Pagano, S. Pirrone, M. F. Rivet and F. Rizzo, eds., Proc. Int. Workshop on Multifragmentation and related topics 2011, Caen, France (2012), vol. 31, 10, http://dx.doi.org/10.1051/epjconf/20123100010.

37. M. Kabtoul, thèse de doctorat, Université de Caen (2013).

38. J. Galin, B. Gatty, D. Guerreau, M. Lefort, X. Tarrago et al., Z. Phys. A 278, 347 (1976).

39. T. H. Chiang, D. Guerreau, P. Auger, J. Galin, B. Gatty et al., Phys. Rev. C 20, 1408 (1979).

40. V. Borrel, B. Gatty, D. Guerreau, J. Galin and D. Jacquet, Z. Phys. A 324, 205 (1986).

41. E. De Filippo, A. Pagano, P. Russotto, F. Amorini, A. Anzalone et al., Phys. Rev. C 86, 014610 (2012).

42. F. Amorini, G. Cardella, G. Giuliani, M. Papa, C. Agodi et al., Phys. Rev. Lett. 102, 112701 (2009).

43. M. B. Tsang, Y. Zhang, P. Danielewicz, M. Famiano, Z. Li et al., Phys. Rev. Lett. 102, 122701 (2009).

44. M. B. Tsang, J. R. Stone, F. Camera, P. Danielewicz, S. Gandolfi et al., Phys. Rev. C 86, 015803 (2012).

45. Z. Y. Sun, M. B. Tsang, W. G. Lynch, G. Verde, F. Amorini et al., Phys. Rev. C 82, 051603 (2010).

46. G. Lehaut, D. Durand, O. Lopez, E. Vient, A. Chbihi et al. (INDRA and ALADIN collaborations), Phys. Rev. Lett. 104, 232701 (2010).

47. D. Durand, B. Tamain and E. Suraud, Nuclear $d y$ namics in the nucleonic regime (Institute of Physics Publishing, London) (2000).

48. J. M. Lattimer and M. Prakash, Science 304, 536 (2004).

49. A. Andronic, J. Łukasik, W. Reisdorf and W. Trautmann, P. Chomaz, F. Gulminelli, W. Trautmann and S. J. Yennello, eds., Dynamics and Thermodynamics 
with nuclear degrees of freedom (Springer), vol. 30 of Eur. Phys. J. A, 31-46 (2006).

50. N. Metropolis, R. Bivins, M. Storm, J. M. Miller, G. Friedlander et al., Phys. Rev. 110, 204 (1958).

51. K. Kikuchi and M. Mawai, Nuclear matter and nuclear reactions (North-Holland Publisher, Amsterdam) (1968).

52. G. Q. Li and R. Machleidt, Phys. Rev. C 48, 1702 (1993).

53. B. Chen, F. Sammarruca and C. A. Bertulani, Phys. Rev. C 87, 054616 (2013); F. Sammarruca, Eur. Phys. J this issue (2013), arXiv:1307.5373[nucl-th].

54. H. Savajols (VAMOS Collaboration), Nucl. Inst. Meth. in Phys. Res. B 204, 146 (2003).

55. S. Pullanhiotan, M. Rejmund, A. Navin, W. Mittig and S. Bhattacharyya, Nucl. Inst. Meth. in Phys. Res. A 593, 343 (2008).

56. S. Pullanhiotan, A. Chatterjee, B. Jacquot, A. Navin and M. Rejmund, Nucl. Inst. Meth. in Phys. Res. B 266, 4148 (2008).

57. A. J. Sierk, Phys. Rev. Lett. 55, 582 (1985).

58. A. Brondi, A. Di Nitto, G. La Rana, R. Moro, P. Nadotchy et al., Proc. II Int. Workshop on Compound Nucleus Reactions (EDP Sciences) (2010), vol. 2, 04002, http://dx.doi.org/10.1051/epjconf/20100204002.

59. R. J. Charity, M. A. McMahan, G. J. Wozniak, R. J. McDonald, L. G. Moretto et al., Nucl. Phys. A 483, 371 (1988).

60. S. Shlomo and J. B. Natowitz, Phys. Rev. C 44, 2878 (1991).

61. G. Nebbia, K. Hagel, D. Fabris, Z. Majka, J. Natowitz et al., Phys. Lett. B 176, 20 (1986).

62. S. I. Al-Quraishi, S. M. Grimes, T. N. Massey and D. A. Resler, Phys. Rev. C 63, 065803 (2001).

63. G. Ademard, J. P. Wieleczko, J. Gomez del Campo, M. La Commara, E.Bonnet et al., Phys. Rev. C 83, 054619 (2011).

64. R. Charity, D. Bowman, Z. Liu, R. McDonald, M. McMahan et al., Nucl. Phys. A 476, 516 (1988).

65. V. E. Viola, K. Kwiatkowski and M. Walker, Phys. Rev. C 31, 1550 (1985).

66. D. Mancusi, R. J. Charity and J. Cugnon, Proc. Int. Workshop on Multifragmentation and related topics 2011, Caen, France (2012), vol. 17, 3, http://dx.doi.org/10.1051/epjconf/20111710003.

67. A. Lalazissis, D. Vretenar and P. Ring, Nucl. Phys. A 679, 481 (2001).

68. P. Marini, thèse de dottorato di ricerca in fisica, Università di Bologna (2009).

69. P. Marini, M. F. Rivet, B. Borderie, N. Le Neindre, A. Chbihi et al. (INDRA collaboration), Proc. II Int. Workshop on Compound Nucleus Reactions (EDP Sciences) (2010), vol. 2, 04003, http://dx.doi.org/10.1051/epjconf/20100204003.

70. H. Gauvin, D. Guerreau, Y. L. Beyec, M. Lefort, F. Plasil et al., Phys. Lett. B 58, 163 (1975).

71. J. P. Coffin, G. Guillaume, A. Fahli, F. Rami, B. Heusch et al., Phys. Rev. C 30, 539 (1984).
72. G. Lehaut, F. Gulminelli and O. Lopez, Phys. Rev. E 81, 051104 (2010).

73. A. Ono, Phys. Rev. C 59, 853 (1999).

74. M. Colonna, Phys. Rev. Lett. 110, 042701 (2013).

75. A. Ono, P. Danielewicz, W. A. Friedman, W. G. Lynch and M. B. Tsang, Phys. Rev. C 70, 041604 (2004).

76. W. D. Myers and W. J. Swiatecki, Nucl. Phys. A 81, 1 (1966); P. Moller, J. R. Nix, W. D. Myers and W. J. Swiatecki, At. Data Nucl. Data Tables 59, 185 (1995); P. Danielewicz, Nucl. Phys. A 727, 233 (2003).

77. F. Chapelle, G. F. Burgio, P. Chomaz and J. Randrup, Nucl. Phys. A 540, 227 (1992).

78. J. Rizzo, P. Chomaz and M. Colonna, Nucl. Phys. A 806, 40 (2008).

79. P. Napolitani and M. Colonna, Phys. Lett. B 726, 382 (2013).

80. P. Napolitani and M. Colonna, J. D. Frankland, A. Pagano, S. Pirrone, M. F. Rivet and F. Rizzo, eds., Proc. Int. Workshop on Multifragmentation and related topics 2011, Caen, France (2012), vol. 31, 27, http://dx.doi.org/10.1051/epjconf/20123100027.

81. M. Colonna, V. Baran, M. Di Toro and H. H. Wolter, Phys. Rev. C 78, 064618 (2008).

82. F. Gagnon-Moisan, thèse de doctorat, Université Paris XI Orsay et Université Laval Québec (2010), http://tel.archives-ouvertes.fr/tel-00491182.

83. R. Bougault, G. Poggi et al., Eur. Phys. J this issue (2013).

84. F. Gulminelli, G. Poggi and G. Verde, Letter of Intent SPIRAL2 GANIL (2006), http://fazia.in2p3.fr/documents/ LoI_SPIRAL2_ThermoDyn_v7.pdf.

85. J. Cornell, ed., Final Report of the EURISOL project, (GANIL), chap. Appendix A : the physics cases (2003), http://pro.ganil-spiral2.eu/eurisol.

86. G. Lehaut, thèse de doctorat, Université de Caen (2009), http://tel.archives-ouvertes.fr/tel-00426878.

87. G. Lehaut, F. Gulminelli and O. Lopez, Phys. Rev. Lett. 102, 142503 (2009).

88. H. Xu, M. B. Tsang, T. X. Liu, X. D. Liu, W. G. Lynch et al., Phys. Rev. Lett. 85, 716 (2000).

89. M. B. Tsang, W. A. Friedman, C. K. Gelbke, W. G. Lynch, G. Verde et al., Phys. Rev. Lett. 86, 5023 (2001).

90. G. A. Souliotis, D. V. Shetty, M. Veselsky, G. Chubarian, L. Trache et al., Phys. Rev. C 68, 024605 (2003).

91. E. Geraci, M. Bruno, M. D'Agostino, E. D. Filippo, A. Pagano et al. (Reverse collaboration), Nucl. Phys. A 732, 173 (2004).

92. A. Le Fèvre, G. Auger, M. L. Begemann-Blaich, N. Bellaize, R. Bittiger et al. (INDRA and ALADIN Collaborations), Phys. Rev. Lett. 94, 162701 (2005).

93. A. Botvina, O. V. Lozhkin and W. Traumann, Phys. Rev. C 65, 044610 (2002).

94. M. B. Tsang, C. K. Gelbke, X. D. Liu, W. G. Lynch, W. P. Tan et al., Phys. Rev. C 64, 054615 (2001). 
95. C. Das and S. Das Gupta, Nucl. Phys. A 812, 149 (2008).

96. G. Chaudhuri, S. D. Gupta and M. Mocko, Nucl. Phys. A 813, 293 (2008).

97. M. B. Tsang, R. Bougault, R. Charity, D. Durand, W. A. Friedman et al., P. Chomaz, F. Gulminelli, W. Trautmann and S. J. Yennello, eds., Dynamics and Thermodynamics with nuclear degrees of freedom (Springer), vol. 30 of Eur. Phys. J. A, 129-139 (2006).

98. J. Richert and P. Wagner, Phys. Rep. 350, 1 (2001).

99. G. A. Souliotis, D. V. Shetty, A. Keksis, E. Bell, M. Jandel et al., Phys. Rev. C 73, 024606 (2006).

100. A. Chbihi, G. Verde, J. D. Frankland, G. Mukherjee and S. Kundu (INDRA collaboration), B. Borderie, J. D. Frankland, A. Pagano, S. Pirrone and F. Rizzo, eds., Proc. Int. Workshop on Multifragmentation and related topics 200\%, Caen, France (2005), 277-285.

101. D. Cugnon and D. L'Hôte, Nucl. Phys. A 397, 519 (1983).

102. J. F. Lecolley, L. Stugge, M. Aboufirassi, A. Badala, B. Bilwes et al., Phys. Lett. B 325, 317 (1994).

103. J. Łukasik, J. Benlliure, V. Métivier, E. Plagnol, B. Tamain et al. (INDRA Collaboration), Phys. Rev. C 55, 1906 (1997).

104. R. Yanez, S. Hudan, R. Alfaro, B. Davin, Y. Larochelle et al., Phys. Rev C 68, 011602 (2003).

105. S. Piantelli, P. R. Maurenzig, A. Olmi, L. Bardelli, A. Bartoli et al., Phys. Rev. C 74, 034609 (2006). 